\title{
A low diffusion MUSCL scheme for LES on unstructured grids
}

Simone Camarri,

Maria Vittoria Salvetti,

Alain Dervieux,

Bruno Koobus

$\mathbf{N}^{\circ} 4512$

July 19, 2002

THÈME 4 



\title{
A low diffusion MUSCL scheme for LES on unstructured grids
}

\author{
Simone Camarri*, \\ Maria Vittoria Salvetti ${ }^{\dagger}$, \\ Alain Dervieux ${ }^{\ddagger}$, \\ Bruno Koobus $\S$
}

Thème 4 - Simulation et optimisation de systèmes complexes

Projet Smash

Rapport de recherche $\mathrm{n}^{\circ} 4512$ - July 19, 2002 - 36 pages

\begin{abstract}
We are interested in the developement of LES methods for compressible flows in complex geometries. Our starting point is a numerical scheme applying to unstructured tetrahedrizations, that is conservative, upwind of MUSCL type and vertex centered. We extend it to a low diffusion version stabilised with six-order derivatives. The new scheme is combined with two LES models, derived from the Smagorinsky model and the dynamic Germano model. The basic test case choosen is the flow around a square cylinder
\end{abstract}

Key-words: Turbulence, large eddy simulation, compressible flow, unstructured mesh

\footnotetext{
* Dipartimento di Ingegneria Aerospaziale, Universita di Pisa, Via Caruso, 56126 Pisa, Italy

$\dagger$ Dipartimento di Ingegneria Aerospaziale, Universita di Pisa, Via Caruso, 56126 Pisa, Italy

‡ INRIA, 2004 Route des Lucioles, BP. 93, 06902 Sophia-Antipolis, France

$\S$ Université de Montpellier II, Département de Mathématiques, CC.051 34095 MONTPELLIER Cedex 5, France and INRIA
} 


\section{Schéma MUSCL à faible diffusion pour la simulation de grandes structures en maillage non-structuré}

Résumé : Ce travail concerne le développement de méthodes de simulation des grandes structures pour les écoulements compressibles dans des géométries complexes. Notre point de départ est un schéma numérique s'applicant à des tétraédrisations non-structurées, qui est conservatif, décentré de type MUSCL, avec des volumes atttachés aux sommets. Nous l'étendons à une version à faible diffusion stabilisée par des dérivées sixièmes. Le nouveau schéma est combiné avec deux modèles sous-grille dérivés de celui de Smagorinsky et du dynamique de Germano. Le cas test d'étude est l'écoulement autour d'un cylindre à section carrée.

Mots-clés : Turbulence, simulation des grandes structures, écoulement compressible, maillage non-structuré 


\section{Contents}

1 Introduction $\quad 3$

2 Numerical Ingredients 4

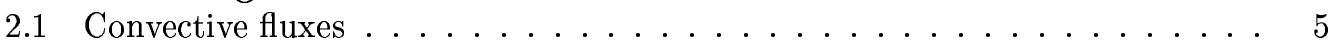

2.1.1 Basic scheme for convective fluxes . . . . . . . . . . . 5

2.1.2 Previous scheme for convective fluxes . . . . . . . . . . 6

2.1.3 New scheme for convective fluxes . . . . . . . . . . . . . 7

2.2 Time advancing . . . . . . . . . . . . . . . . . . . . 9

2.3 Dissipation properties: V4 vs V6 . . . . . . . . . . . . . . 10

2.4 LES approach to turbulence . . . . . . . . . . . . . . . . . . 12

2.4 .1 Smagorinsky model . . . . . . . . . . . . . . . . . . . . 12

2.4 .2 Dynamic model . . . . . . . . . . . . . . . . . . 14

3 Tests 14

3.1 Test-case and simulations . . . . . . . . . . . . . . . . . 14

3.2 Results . . . . . . . . . . . . . . . . . . . . 16

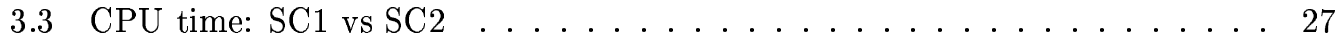

4 Conclusions $\quad 31$

\section{Introduction}

Large-eddy simulation (LES) has been successfully used in the last years to simulate different turbulent flows. However, up to now most of the simulations reported in the literature are limited to simple geometries and moderate Reynolds numbers. In the perspective of application of LES in an industrial context, new problems arise.

As far as computational domain discretization is concerned, structured grids, classically used in LES, are extremely difficult to be generated around complex geometries. Unstructured grids offer an effective alternative; however, only few examples of LES on unstructured grids are reported in the literature ([4], [5], [8], [14], [15], [16], [23], [25]). Thus, the capabilities of LES on this type of grids need to be investigated more systematically.

The use of unstructured grids and the need of limiting the computational costs naturally lead to low-order (second-order) co-located schemes. Indeed, higher-order mixed finiteelement methods are expensive for unstructured grids. Although the use of second-order schemes in LES is still a controversial point, successful second-order accurate LES have been documented in the literature for a wide variety of flows. In our opinion, the most critical point with co-located schemes is the need of numerical dissipation. Indeed, pressure, as well as the remaining flow parameters, is stabilized by the upwinding of the scheme and non-physical pressure oscillations are obtained if the numerical viscosity is too low. In some studies in the literature, it is claimed that the numerical dissipation given by monotone flux 
treatments may be an accurate SGS model; see [25] for an application to unstructured grids. However, we remark that this family of methods seem to need a much larger number of nodes in the mesh for a given prediction quality. When monotone schemes are combined with a classical LES model, they can interact unfavorably with it, and significantly deteriorate the results (see, for instance, [12]). Thus, it appears that the effects of numerical dissipation and SGS models should be separated as much as possible. In all the previous applications of LES to unstructured grids ([4], [5], [8], [14], [15], [16], [23], [25]), numerical dissipation is provided by second-order spatial derivatives, either through Petrov-Galerkin upwinding or through TVD limiters. However, a third order Taylor-Galerkin scheme applicable to LES on unstructured grids has been proposed in Ref. [6].

In a previous work ([2] and [3]), we used a MUSCL upwind scheme, which involves a dissipation built as a fourth-order spatial derivative of the flow variables and no TVD limiters. Fourier analysis clearly shows that such a dissipation has a much more localized effect on high frequencies than stabilizations based on second-order derivatives. Moreover, a key coefficient $\left(\gamma_{s}\right)$ permits to tune numerical dissipation to the smallest amount required to stabilize the simulation. This possibility to tune the numerical viscosity level is an important advantage with respect to existing methods involving fourth-order derivative as stabilization (such as Ref. [6]). In this way we can reduce the interaction between numerical dissipation, which damps in priority the highest frequencies, in particular those for which the phase error is too large and can produce oscillations, and SGS modeling, which should reproduce the effects of unresolved frequencies on all the resolved ones, is reduced. This approach was appraised in the LES of the flow around a square cylinder at $R e=22000$ on unstructured grids and the accuracy of the results obtained with the lowest values of $\gamma_{s}$ allowed by the scheme stability was comparable to that of other LES carried out with centered schemes on more resolved structured grids ([27], [30]).

In the present study, we investigate a MUSCL scheme in which stabilization is obtained through numerical diffusion based on sixth-order derivatives (as proposed in [7]), in order to further enhance the complementarity between the SGS model and the MUSCL stabilization and to further reduce their competition. As in the previous scheme, the coefficient $\gamma_{s}$ directly controls the amount of numerical dissipation. The proposed approach is used in large-eddy simulations of the square cylinder test-case and results are compared with those previously obtained with the fourth-order derivative upwinding and with experimental and LES data in the literature.

\section{Numerical Ingredients}

The numerical code considered herein (AERO) is a Navier-Stokes solver for Newtonian, compressible and three-dimensional flows [10]. AERO is a prototype for demonstrating innovative methods in fluid-structure interaction. It has been intensively applied to various industrial configurations (see, for instance, [17], [18], [29]). It employs unstructured grids for description of complex moving and possibly deforming geometries. A mixed finite- 
volume/finite-element method is used for space discretization. The finite-volume formulation is used for the convective terms and finite-elements for the diffusive terms.

The Roe scheme [28] represents the basic upwind component for the numerical evaluation of the convective fluxes and a MUSCL linear reconstruction method ("Monotone Upwind Schemes for Conservation Laws"), introduced by Van Leer [32], is employed to increase the order of accuracy of the Roe scheme.

Either implicit or explicit second-order schemes can be used to advance the equations in time by a line method, i.e. time and space are treated separately.

The spatial discretization of convective terms is described in the following section while, for other details on AERO solver, we refer to Ref. [10].

\subsection{Convective fluxes}

\subsubsection{Basic scheme for convective fluxes}

Convective fluxes are discretized by a finite-volume approach, in which the fluxes through the cell boundaries must be evaluated:

$$
\sum_{j=n e i g(i)} \int_{\partial C_{i j}} \mathcal{F}(W, \vec{n}) d \sigma
$$

where $\partial C_{i j}$ is the boundary between cells $C_{i}$ and $C_{j}$, and $\vec{n}$ is the outer normal to the cell $C_{i}$ (see Fig. 1). The Roe scheme [28] is the basic upwinding component for the approximation of the convective fluxes:

$$
\int_{\partial C_{i j}} \mathcal{F}(W, \vec{n}) d \sigma \simeq \Phi^{R}\left(W_{i}, W_{j}, \overrightarrow{\nu_{i j}}\right)
$$

in which $\nu_{i j}$ is defined as:

$$
\nu_{i j}=\int_{\partial C_{i j}} \vec{n} d \sigma
$$

Thus, the numerical fluxes are evaluated as follows:

$$
\begin{aligned}
& \Phi^{R}\left(W_{i}, W_{j}, \overrightarrow{\nu_{i j}}\right)=\frac{\mathcal{F}\left(W_{i}, \overrightarrow{\nu_{i j}}\right)+\mathcal{F}\left(W_{j}, \overrightarrow{\nu_{i j}}\right)}{2}-\gamma_{s} d^{R}\left(W_{i}, W_{j}, \overrightarrow{\nu_{i j}}\right) \\
& d^{R}\left(W_{i}, W_{j}, \overrightarrow{\nu_{i j}}\right)=\left|\mathcal{R}\left(W_{i}, W_{j}, \nu_{i j}\right)\right| \frac{W_{j}-W_{i}}{2}
\end{aligned}
$$

where $W_{k}$ is the solution vector at the $k$-th node and $\mathcal{R}$ is the Roe matrix:

$$
\mathcal{R}\left(W_{i}, W_{j}, \nu_{i j}\right)=\frac{\partial \mathcal{F}}{\partial W}\left(\widehat{W}, \nu_{i j}\right)
$$

The term $\widehat{W}$ is the Roe average [28] of $W_{i}$ and $W_{j}$. Note that, in (4), a parameter $\gamma_{s} \in[0,1]$

$\mathrm{RR} \mathrm{n}^{\circ} 4512$ 


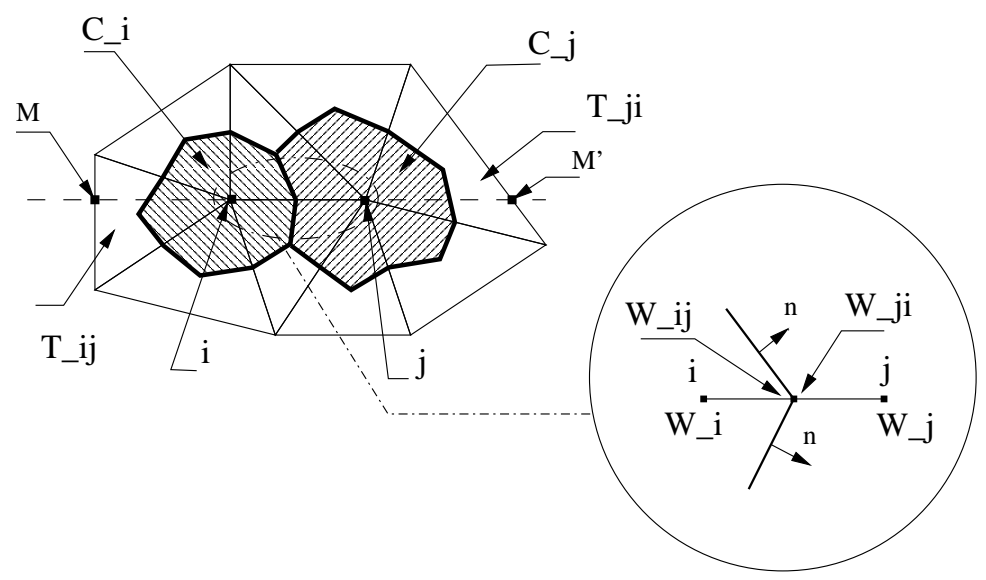

Figure 1: A 2D mesh: cells, elements, notation. The extension to the 3D case is straighforward.

has been introduced, which directly controls the upwinding of the scheme. The classical Roe scheme [28] is obtained as a particular case by imposing $\gamma_{s}=1$.

The spatial accuracy of this scheme is only first order. The MUSCL reconstruction method ("Monotone Upwind Schemes for Conservation Laws"), introduced by Van Leer [32], is therefore employed to increase the order of accuracy. The basic idea is to express the Roe flux as a function of the extrapolated values of $W$ at the interface between the two cells $C_{i}$ $\left(W_{i j}\right)$ and $C_{j}\left(W_{j i}\right)$ :

$$
\begin{aligned}
& \int_{\partial C_{i j}} \mathcal{F}(W, \vec{n}) d \sigma \simeq \Phi^{R}\left(W_{i j}, W_{j i}, \overrightarrow{\nu_{i j}}\right), \\
& W_{i j}=W_{i}+\frac{1}{2}(\vec{\nabla} W)_{i j} \cdot \overrightarrow{i j} \\
& W_{j i}=W_{j}-\frac{1}{2}(\vec{\nabla} W)_{j i} \cdot \overrightarrow{i j}
\end{aligned}
$$

\subsubsection{Previous scheme for convective fluxes}

In the previous approach, a scheme was used in which the numerical viscosity was made by fourth-order derivatives (V4). In this case, the "slopes" $(\vec{\nabla} W)_{i j}$ and $(\vec{\nabla} W)_{j i}$ are obtained 
by a $\beta$-scheme, which combines together centered and upwind gradients:

$$
\left\{\begin{array}{l}
(\vec{\nabla} W)_{i j} \cdot \overrightarrow{i j}=(1-\beta)(\vec{\nabla} W)_{i j}^{C} \cdot \overrightarrow{i j}+\beta(\vec{\nabla} W)_{i j}^{D} \cdot \overrightarrow{i j} \\
(\vec{\nabla} W)_{j i} \cdot \overrightarrow{i j}=(1-\beta)(\vec{\nabla} W)_{i j}^{C} \cdot \overrightarrow{i j}+\beta(\vec{\nabla} W)_{i j}^{U} \cdot \overrightarrow{i j}
\end{array}\right.
$$

The term $(\vec{\nabla} W)_{i j}^{C}$ is the centered gradient, defined as $(\vec{\nabla} W)_{i j}^{C} \cdot \overrightarrow{i j}=W_{j}-W_{i}$. The upwind $(\vec{\nabla} W)_{i j}^{U}$ and downwind $(\vec{\nabla} W)_{i j}^{D}$ gradients are computed respectively on the upwind $\left(T_{j i}\right)$ and downwind $\left(T_{i j}\right)$ tetrahedra associated with the edge $\overrightarrow{i j}$ (see Fig. 1 for the 2D case and Fig. 2 for the 3D case). In particular, $(\vec{\nabla} W)_{i j}^{U}=\left.\vec{\nabla} W\right|_{T_{j i}}$ and $(\vec{\nabla} W)_{i j}^{D}=\left.\vec{\nabla} W\right|_{T_{i j}}$ where $\left.\vec{\nabla} W\right|_{T}=\left.\sum_{k \in T} W_{k} \vec{\nabla} \Phi_{k}\right|_{T}$ is the P1-Galerkin gradient on the triangle $T$.

A classical (spatial) truncation error analysis of the previous scheme, for the 2D linear advection equation discretized on a regular triangular grid of Friedrichs-Keller type, shows that, when $\beta=1 / 3$, the leading error term is a third-order dissipation error, formed of fourth-order space derivatives of the flow variables. Note, however, that this analysis does not take into account either the non-linearity of the hyperbolic terms in the Navier-Stokes equations or the possible non-uniformity of the grid. The scheme presented in this section, with $\beta=1 / 3$, will be denoted in the following as SC1.

\subsubsection{New scheme for convective fluxes}

The new discretization of convective fluxes is still based on the Roe scheme used together with a MUSCL reconstruction method, but the gradients $(\vec{\nabla} W)_{i j}$ and $(\vec{\nabla} W)_{j i}$ are now estimated as follows:

$$
\begin{aligned}
(\vec{\nabla} W)_{i j} \cdot \overrightarrow{i j}= & (1-\beta)(\vec{\nabla} W)_{i j}^{C} \cdot \overrightarrow{i j}+\beta(\vec{\nabla} W)_{i j}^{U} \cdot \overrightarrow{i j} \\
& +\xi_{c}\left[(\vec{\nabla} W)_{i j}^{U} \cdot \overrightarrow{i j}-2(\vec{\nabla} W)_{i j}^{C} \cdot \overrightarrow{i j}+(\vec{\nabla} W)_{i j}^{D} \cdot \overrightarrow{i j}\right] \\
& +\xi_{d}\left[(\vec{\nabla} W)_{M} \cdot \overrightarrow{i j}-2(\vec{\nabla} W)_{i} \cdot \overrightarrow{i j}+(\vec{\nabla} W)_{j} \cdot \overrightarrow{i j}\right], \\
(\vec{\nabla} W)_{j i} \cdot \overrightarrow{i j}= & (1-\beta)(\vec{\nabla} W)_{j i}^{C} \cdot \overrightarrow{i j}+\beta(\vec{\nabla} W)_{j i}^{U} \cdot \overrightarrow{i j} \\
& +\xi_{c}\left[(\vec{\nabla} W)_{j i}^{U} \cdot \overrightarrow{i j}-2(\vec{\nabla} W)_{j i}^{C} \cdot \overrightarrow{i j}+(\vec{\nabla} W)_{j i}^{D} \cdot \overrightarrow{i j}\right] \\
& +\xi_{d}\left[(\vec{\nabla} W)_{M^{\prime}} \cdot \overrightarrow{i j}-2(\vec{\nabla} W)_{i} \cdot \overrightarrow{i j}+(\vec{\nabla} W)_{j} \cdot \overrightarrow{i j}\right] .
\end{aligned}
$$

where $(\vec{\nabla} W)_{i}$ and $(\vec{\nabla} W)_{j}$ are the nodal gradients respectively on the nodes $i$ and $j$. The nodal gradient $(\vec{\nabla} W)_{i}$ is calculated as the average of the gradients on tetrahedra $T \in C_{i}$ 
Tij

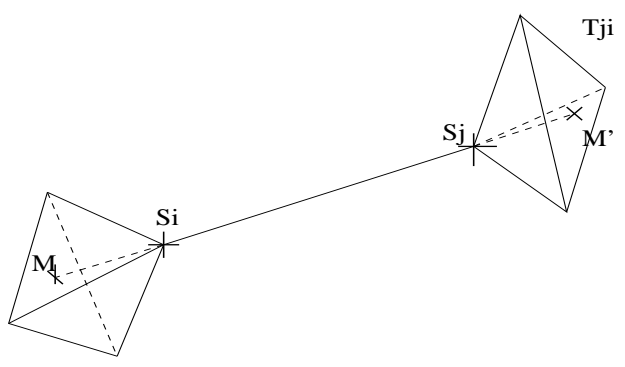

Figure 2: Sketch of points and elements involved in the computation of gradients

\begin{tabular}{|c|c|c|c|c|c|}
\hline Scheme & $\gamma_{s}$ & $\beta$ & $\xi^{c}$ & $\xi^{d}$ & order \\
\hline SC2 & ] $0,1]$ & $1 / 3$ & $-1 / 30$ & $-2 / 15$ & 5 \\
SC3 & {$[0,1]$} & $1 / 3$ & 0 & $-1 / 6$ & 4 \\
SC4 & 0 & $1 / 3$ & $-1 / 30$ & $-2 / 15$ & 6 \\
\hline
\end{tabular}

Table 1: V6 Schemes

having the node $i$ as a vertex:

$$
(\vec{\nabla} W)_{i}=\frac{1}{\operatorname{Vol}\left(C_{i}\right)} \sum_{T \in C_{i}} \frac{\operatorname{Vol}(T)}{3} \sum_{k \in T} W_{k} \vec{\nabla} \Phi_{k}^{T}
$$

The term $(\vec{\nabla} W)_{M}$ is the gradient at the point $M$ of Fig. 1 (2D case) and Fig. 2 (3D case). This last gradient is computed by interpolation of the nodal gradient values at the nodes contained in the face opposite to $i$ in the upwind tetrahedron $T_{i j}$ (see Fig. 1 and Fig. 2). The term $(\vec{\nabla} W)_{M^{\prime}}$ is the gradient at the point $M^{\prime}$ (see Fig. 1 and Fig. 2) and it is evaluated in the same way as $(\vec{\nabla} W)_{M}$.

The coefficients $\beta, \xi^{c}$ and $\xi^{d}$ are parameters that control the combination of fully upwind and centered slopes. If $\beta=1 / 3$ the resulting scheme is at least third-order accurate. Then, the parameters $\xi^{c}$ and $\xi^{d}$ can be tuned in order to increase the accuracy of the scheme. In Tab. 1, three examples are reported. The scheme SC2 is fifth-order accurate and it has a dissipative leading error proportional to the sixth-order derivatives. The scheme SC3 is fourth-order accurate and it has a leading error of dispersive type that is proportional to the fifth-order derivatives; consequently, it also provides a numerical viscosity proportional to the sixth-order derivatives. Finally, the scheme SC4, obtained from SC2 imposing $\gamma_{s}=0$, corresponds to a centered scheme (see Eq. (4)) and it is sixth-order accurate. Note that the results of Tab. 1 come from a classical (spatial) truncation error analysis for the 2D linear advection equation, discretized on a regular triangular grid of Friedrichs-Keller type. In a general non-linear case, the schemes of Tab. 1 still have dissipation made of sixth-order

INRIA 


\begin{tabular}{|c|c|c|c|c|c|c|}
\hline scheme & $\alpha_{1}$ & $\alpha_{2}$ & $\alpha_{3}$ & $\alpha_{4}$ & $\alpha_{5}$ & $\alpha_{6}$ \\
\hline \hline $\mathrm{R} 1$ & 1 & $1 / 2$ & 0.2766 & 0.11 & - & - \\
\hline $\mathrm{R} 2$ & 1 & $1 / 2$ & $1 / 3$ & $1 / 4$ & - & - \\
\hline $\mathrm{R} 3$ & 1 & $1 / 2$ & $1 / 3$ & $1 / 4$ & $1 / 5$ & $1 / 6$ \\
\hline
\end{tabular}

Table 2: Coefficients for low-stockage Runge Kutta algorithms.

derivatives, but they are only second-order accurate. (The scheme SC2 of Tab. 1 has been used in the simulations presented in the following.)

\section{$2.2 \quad$ Time advancing}

Either implicit or explicit schemes can be used to advance the equations in time by a line method, i.e. time and space are treated separately. Once the equations have been discretized in space, a set of ordinary differential equations in time is obtained:

$$
W_{t}+\Psi(W)=0
$$

In the explicit case a N-stage low-storage Runge Kutta algorithm is used for the discretization of Eq. (15):

$$
\left\{\begin{array}{l}
W^{(0)}=W^{(n)} \\
W^{(k)}=W^{(0)}+\Delta t \alpha_{k} \Psi\left(W^{(k-1)}\right), \quad k=1, \cdots, N \\
W^{(n+1)}=W^{(N)}
\end{array}\right.
$$

Different schemes can be obtained by varying the number of stages $N$ and the coefficients $\alpha_{k}$, as shown in Tab. 2.

The V6 family of schemes (Tab. 1) enjoys rather good stability properties, with stable CFL number larger than unity, when an explicit Runge Kutta algorithm is used in time. This is shown in Ref. [7] for a linear 1D convection equation when the 6-stage Runge Kutta scheme R3 of Tab. 2 is used in time.

In the simulations presented here, the 4-stage algorithm R1 of Tab. 2 was used. The stability of the schemes of Tab. 1, when used together with the Runge Kutta algorithms of Tab. 2, has been appraised by performing tests directly on the code. The results are reported in Appendix I.

In the implicit case, a second-order backward differencing scheme is applied [26], which involves an explicit time derivative expressed only as a spatial residual, so that it does not depend on the time step length The globally resulting method is linearly unconditionally stable, it is second order accurate in space and time and allows stable calculations to be carried out on very heterogeneous grids (with locally very small cells) and for a large range of Mach numbers.

Although it was shown in Ref. [2] that implicit time advancing can be advantageous when used for coarse LES simulations, it was not used here because the test case selected 
Camarri, Salvetti, Dervieux, Koobus,

requires the use of periodic boundary conditions, which have only been implemented only for the explicit time advancing on AERO.

\subsection{Dissipation properties: V4 vs V6}

The schemes SC1 and SC2 provide a diffusion based respectively on fourth-order and sixthorder space derivatives. Consequently, numerical viscosity in $\mathrm{SC} 2$ is more concentrated on high-frequency components than in SC1. This can be shown by performing a von Neumann stability analysis of these schemes when the algorithm R2 in Tab. 2 is used for time discretization. A mono-dimensional linear convection equation can be considered to this purpose:

$$
u_{t}+c u_{x}=0, \quad c>0
$$

Eq. (17) is discretized in space using the corresponding schemes of SC1 (SC1-1D) and SC2 (SC2-1D) for the mono-dimensional case (see Ref. [7]). An uniform discretization both in space $\left(x_{j}=j \cdot \Delta x\right)$ and in time $\left(t_{n}=n \cdot \Delta t\right)$ is used. A Fourier mode in space with a period $T_{k}=2 \pi / \Theta_{k} \Delta x$ is considered as the solution of Eq. (17) at time $t_{n}$ :

$$
u\left(x_{j}, t_{n}\right)=u_{j}^{(n)}=\hat{u}_{k}^{(n)} e^{i\left(j \Theta_{k}\right)} .
$$

The solution $u_{j}^{(n)}$ of Eq. (18) is then introduced in the discretized form of the equation Eq. (17). Since Eq. (17) is linear, we obtain:

$$
\hat{u}_{k}^{(n+1)}=\left[g\left(\Theta_{k}\right)\right] \hat{u}_{k}^{(n)}
$$

The amplification factor $\left|g\left(\Theta_{k}\right)\right|$ and the phase angle of $g\left(\Theta_{k}\right)$ represents the effect of the numerical scheme respectively on the amplitude and on the phase of the Fourier mode having period $T_{k}=2 \pi / \Theta_{k} \Delta x$. Note that, since the Fourier mode is sampled in space at the discrete points $x_{j}$, with spacing $\Delta x$, the following condition needs to be verified in order to guarantee that the mode is properly represented:

$$
T_{k}>2 \Delta x \Rightarrow \Theta_{k}<\pi \text {. }
$$

When a mode $h$ with $\Theta_{h}=\pi+\phi$ is sampled at points $x_{j}$, it is equivalent to a mode $k$ with $\Theta_{k}=\pi-\phi$, due to aliasing errors. Consequently, the amplification factor and the phase error plots have been plotted only for $\Theta_{k} \in[0, \pi]$.

The amplification factor and the phase error curve for the schemes SC1-1D and SC2-1D are shown respectively in Fig. 3 and Fig. 4, for a CFL number varying in the range [0.8, 1.2]. Fig. 3 and Fig. 4 shows that both SC1-1D and SC2-1D concentrate damping on high frequency components. However, it is possible to notice in Fig 5, where the amplification factors of both schemes for $C F L=1$ are plotted together, that when SC2-1D is used, the low frequency components are significantly less damped by numerical dissipation and there is a sharper separation between the scales affected by numerical viscosity and the remaining ones. 

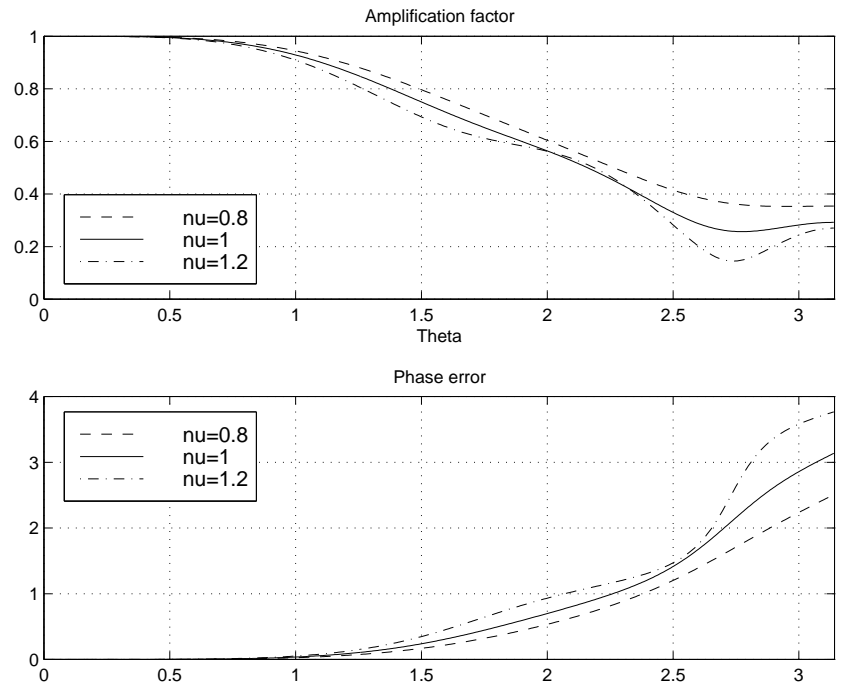

Figure 3: Amplification factor and phase error at different CFL numbers (nu); scheme SC1$1 \mathrm{D}$.
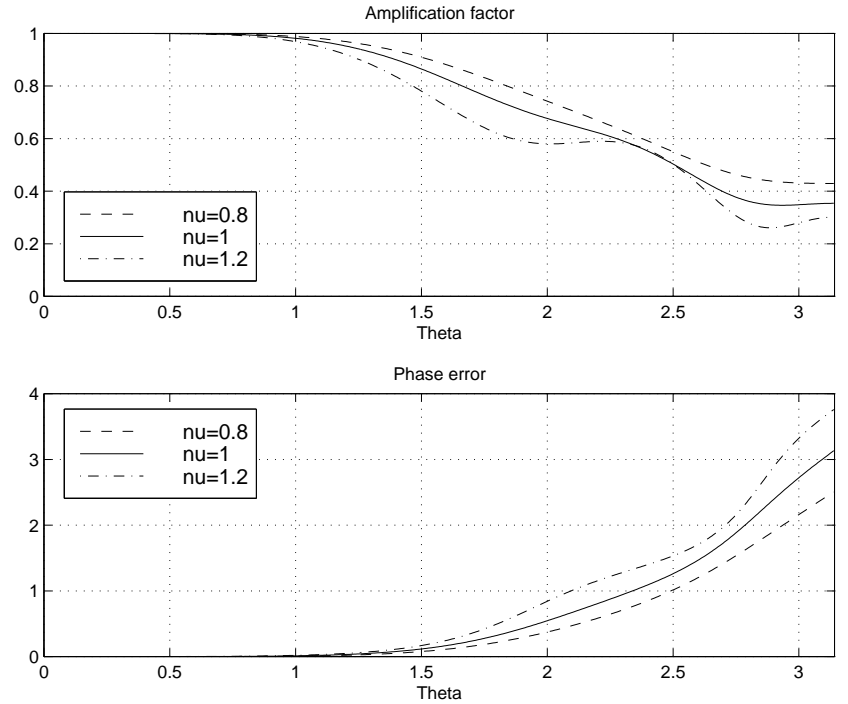

Figure 4: Amplification factor and phase error at different CFL numbers (nu); scheme SC2$1 \mathrm{D}$.

$\mathrm{RR} \mathrm{n}^{\circ} 4512$ 


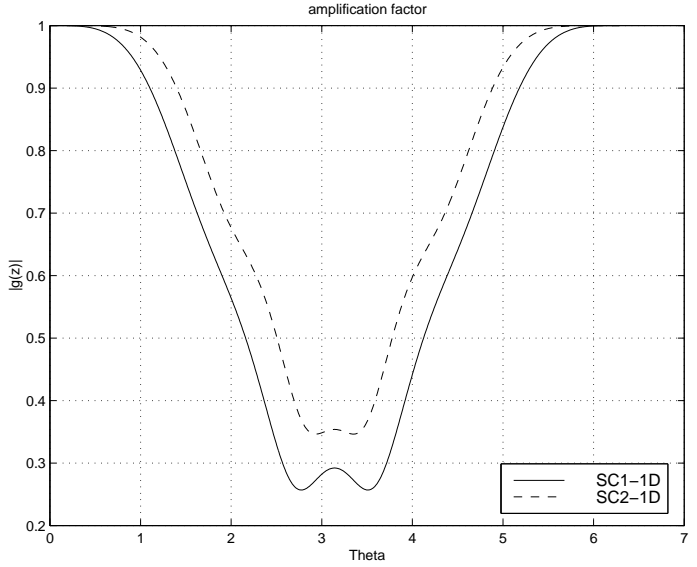

(a) amplification factors

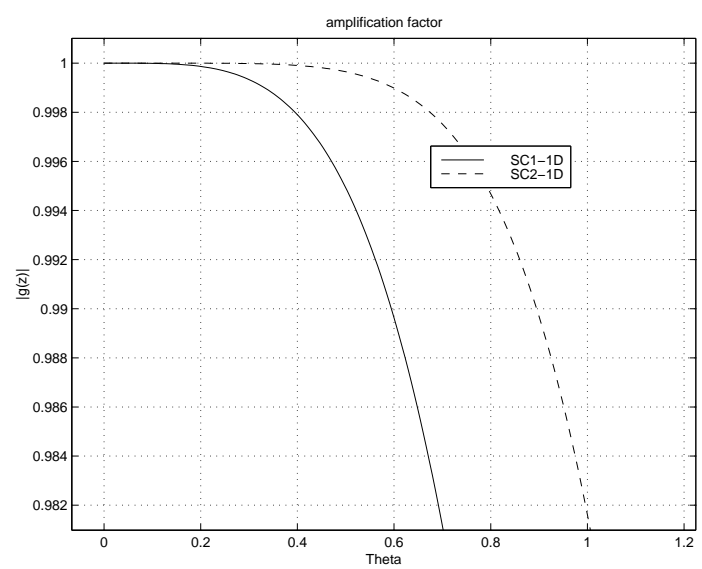

(b) amplification factors (detail)

Figure 5: Amplification factors for SC1-1D and SC2-1D $(\mathrm{nu}=1)$

Phase error has a behavior similar to the amplification factor, as it can be seen by comparing Fig. 3 and Fig. 4. Indeed, in SC2-1D dispersion error is more concentrated on high frequencies since it is proportional to the seventh-order space derivatives, while in SC1-1D it is made of fifth-order derivatives. This is put in evidence in Fig. 6, where the phase error of both schemes for $C F L=1$ are plotted together.

\subsection{LES approach to turbulence}

The LES (Large Eddy Simulation) approach consists in filtering in space the Navier-Stokes equations, in order to get rid of the high frequency fluctuations, and in simulating directly only the filtered flow. Due to the non-linearity of the problem, the filtered equations contain some unknown terms which represent the effect of the eliminated fluctuations on the filtered flow. These terms need to be modeled.

Implicit filtering is used in AERO, i.e. the numerical discretization of the equations is considered as a filter operator. Two closure models for the LES approach are used in AERO: the Smagorinsky model and its dynamic version.

\subsubsection{Smagorinsky model}

The extension of the Smagorinsky model to compressible flows [19] adopted in AERO is intended to be used to study flows at high Reynolds numbers and such that low compressibility effects are present in the SGS fluctuations. In addition, we assume that heat transfer 


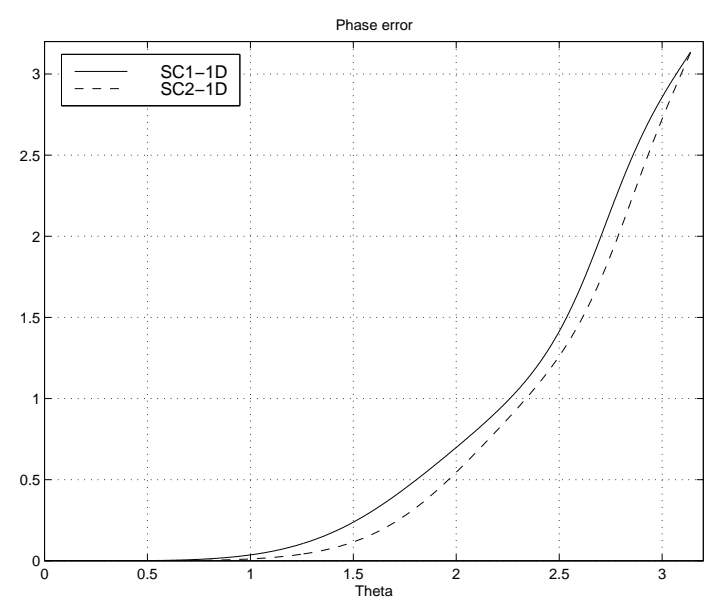

(a) Phase error

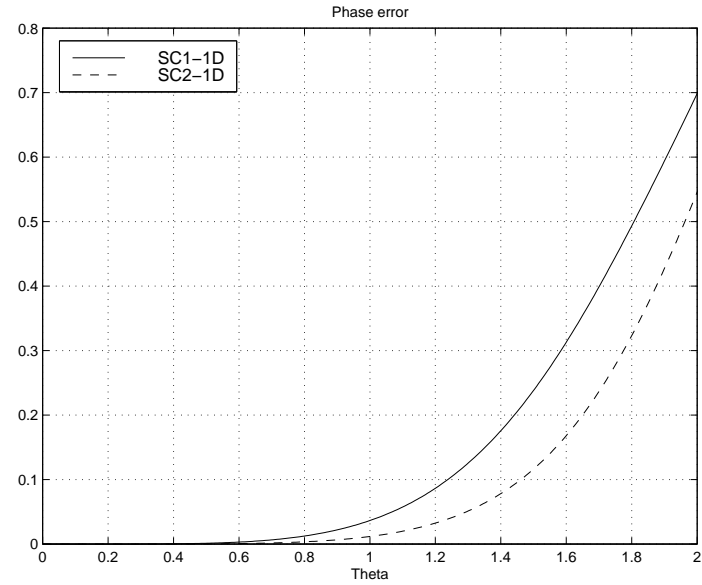

(b) Phase error (detail)

Figure 6: Phase errors for SC1-1D and SC2-1D $(\mathrm{nu}=1)$

and temperature gradients are moderate. Thus, the retained SGS term in the momentum equation is the classical SGS stress tensor:

$$
M_{i j}=\overline{\rho u_{i} u_{j}}-\bar{\rho} \tilde{u}_{i} \tilde{u}_{j},
$$

where the over-line denotes the grid filter and the tilde the density-weighted Favre filter $(\tilde{f}=(\overline{\rho f}) /(\bar{\rho}))$. The isotropic part of $M_{i j}$ can be neglected under the assumption of low compressibility effects in the SGS fluctuations [9]. The deviatoric part, $T_{i j}$, may be expressed by an eddy viscosity term, in accordance with the Smagorinsky model extended to compressible flows ([19]):

$$
\begin{aligned}
& T_{i j}=-2 \mu_{s g s}\left(\widetilde{S_{i j}}-\frac{1}{3} \widetilde{S_{k k}}\right), \\
& \mu_{s g s}=\bar{\rho}\left(C_{s} \Delta\right)^{2}|\widetilde{S}|,
\end{aligned}
$$

$\widetilde{S_{i j}}$ being the resolved strain tensor, $\mu_{\text {sgs }}$ the SGS viscosity, $\Delta$ the filter width, $C_{s}$ a constant

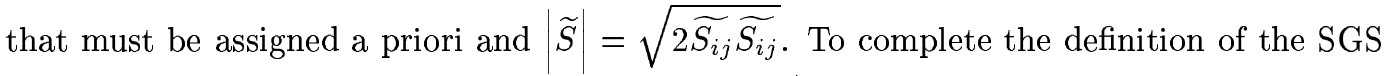
viscosity, the grid filter width must be specified. Although it is not trivial to define the width of the filter corresponding to the numerical discretization on unstructured grids, the following expression has been employed here for each grid element $l$ :

$$
\Delta^{(l)}=\max _{i=1, . ., 6}\left(\Delta_{i}^{(l)}\right)
$$


in which $\Delta_{i}^{(l)}$ is the length of the $i$-th side of the $l$-th element.

In the energy equation, the effect of the SGS fluctuations has been modeled by the introduction of a constant SGS Prandtl number to be assigned a-priori:

$$
\operatorname{Pr}_{s g s}=C_{p} \frac{\mu_{s g s}}{K_{s g s}}
$$

where $K_{s g s}$ is the SGS conductivity coefficient; it takes into account the diffusion of total energy caused by the SGS fluctuations. In the filtered energy equation, the term $K_{s g s}$ is added to the molecular conductivity coefficient.

\subsubsection{Dynamic model}

The dynamic version of the Smagorinsky model has also been considered. The dynamic procedure proposed by Germano [13] is applied to the compressible Smagorinsky model described in Sec. 2.4.1. In this way, the coefficient that must be assigned a-priori in the Smagorinsky model $\left(C_{s}\right)$ is computed as a function of space at each time step. We chose to dynamically compute $\left(C_{s} \Delta\right)^{2}$ instead of $C_{s}^{2}$, as in the classical dynamic model, to avoid the indetermination in the definition of the filter width. The test filter used here consists in P1-averaging the flow variables on all the elements having a given node as a vertex. Thus, the ratio $\frac{\widehat{\Delta}}{\Delta}(\widehat{\Delta}$ being the test filter width), which is the only quantity to be assigned a priori in the dynamic model, is defined on each node as: $\frac{\widehat{\Delta}}{\Delta}=\sqrt[3]{N}$, where $N$ is the number of elements having the node as a vertex. A local smoothing is applied to avoid unphysical oscillations of $\left(C_{s} \Delta\right)^{2}$ (see [3]).

For more details on the LES approach implemented in AERO, see Ref. [3].

\section{Tests}

\subsection{Test-case and simulations}

The flow around a square cylinder at $R e=2.2 \times 10^{4}$ was simulated. This flow was investigated experimentally ([1], [20], [21], [22], [24]); LES results are also available in the literature ([11], [27], [30]). The simulations presented here were performed at a Mach number $M=0.1$, in order to have negligible compressibility effects (experiments were performed for incompressible flow). The flow conditions of the experiments and of the presented simulations are reported in Tab. 3.

The computational domain and the boundary conditions used are represented in Fig. 7. Boundary conditions based on Steger-Warming decomposition [31] are used at the inflow and at the outflow surfaces. On the side surfaces free-slip is imposed and the flow is assumed to be periodic in spanwise direction. Approximate boundary conditions based on the Reichardt wall-law are used on the cylinder surface. For details on the use of LES with Reichardt wall-law see Ref. [2] and Ref. [3]. 


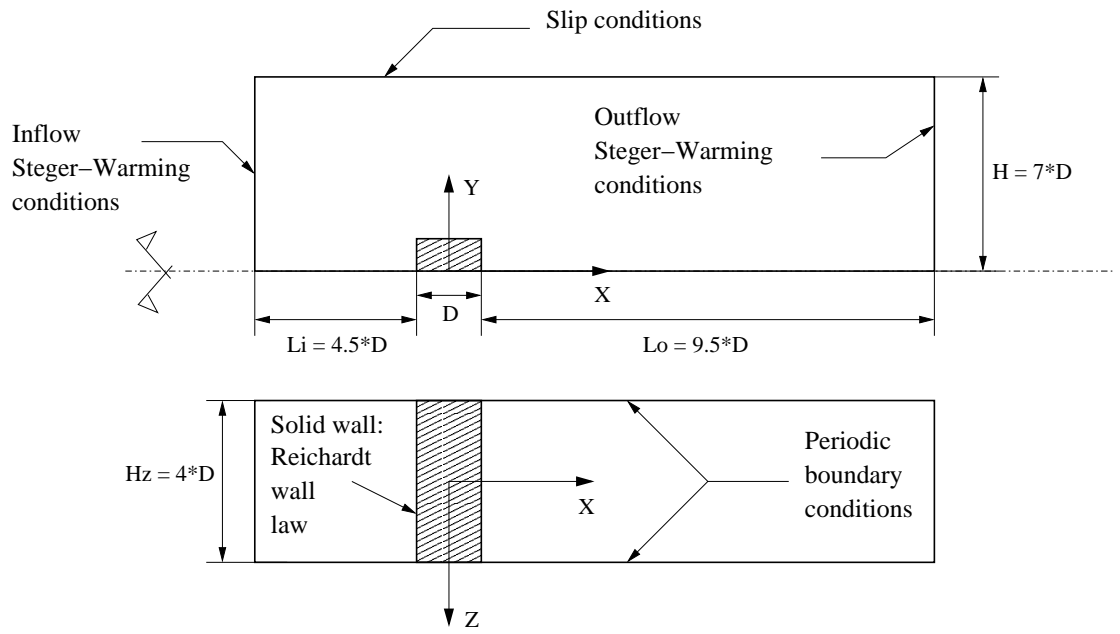

Figure 7: Computatinal domain.

Two different unstructured grids were used, a coarse one (GR1), with about $10^{5}$ nodes and $6 \times 10^{5}$ elements, and a more refined one (GR2), using around $2 \times 10^{5}$ nodes and $1.1 \times 10^{6}$ elements. In both grids, approximately 32 nodes are used in the spanwise direction within the wake region, which corresponds to a spanwise resolution $\Delta z \simeq 0.125 D$. The average distance of the first layer of nodes from the cylinder surface is around $6.0 \cdot 10^{-2} \mathrm{D}$ in GR1 and $4.5 \cdot 10^{-2}$ in GR2. This corresponds approximately to $y^{+} \in[10,100]$ for GR1 and $y^{+} \in[8,100]$ for GR2.

Simulations were performed on GR1 and GR2 using both SGS models described previously (see Sec. 2.4), different values of upwinding $\gamma_{s}$ and the schemes SC1 (Sec. 2.1.1) and SC2 (Sec. 2.1.3) for the convective fluxes discretization. For all the simulations, time advancing was carried out by the 4-stage Runge Kutta algorithm R1 of Tab. 2, with a max-

\begin{tabular}{|c|c|c|c|c|c|c|}
\hline Experiments & $R e / 10^{4}$ & Blockage & $\begin{array}{c}\text { Free stream } \\
\text { turbulence (\%) }\end{array}$ & $\begin{array}{c}\text { Aspect } \\
\text { Ratio }\end{array}$ & $\begin{array}{c}\text { End } \\
\text { plates }\end{array}$ & Corrections \\
\hline Ref. [22, 21] & 2.2 & 7.0 & 2 & 9.75 & No & No \\
\hline Ref. [1] & $0.58 \div 3.2$ & 5.5 & 0.04 & $\simeq 17$ & Yes & No \\
\hline Ref. [24] & 1.3 & $\leq 5$ & 0.06 & $\geq 17$ & Yes & No \\
\hline Ref. [20] & 3.4 & 5 & 0.5 & 9.2 & Yes & Yes \\
\hline \hline Simulations & 2.2 & 7 & 0 & $\infty$ & No & No \\
\hline
\end{tabular}

Table 3: Main parameters in experiments and present simulations. Corrections, if present, are made to account for blockage effects (see cited references for details). 
imum CFL number equal to 0.85. The simulations parameters are summarized in Tab. 4.

\begin{tabular}{llccc}
\hline Simulation & grid & SGS model & $\gamma_{s}$ & Convective fluxes \\
\hline SM1 & GR1 & Smag. $\left(C_{s}=0.1\right)$ & 0.05 & SC1 \\
SM2 & GR1 & Smag. $\left(C_{s}=0.1\right)$ & 0.05 & SC2 \\
SM3 & GR2 & Smag. $\left(C_{s}=0.1\right)$ & 0.05 & SC2 \\
DM1 & GR1 & Dynamic & 0.05 & SC1 \\
DM2 & GR1 & Dynamic & 0.05 & SC2 \\
DM3 & GR1 & Dynamic & 0.1 & SC1 \\
DM4 & GR1 & Dynamic & 0.1 & SC2 \\
DM5 & GR2 & Dynamic & 0.05 & SC2 \\
\hline
\end{tabular}

Table 4: Summary of the simulations.

\subsection{Results}

The main bulk coefficients obtained in the simulations are presented in Tab. 5, together with results from other LES simulations available in the literature ([11], [27], [30]). Available experimental data are also reported in the table ([1], [20], [21], [22], [24]).

\begin{tabular}{lccccc}
\hline LES and experiments & $C_{l}^{\prime}$ & $\overline{C_{d}}$ & $C_{d}^{\prime}$ & $l_{r}$ & $S t$ \\
\hline SM1 & 0.79 & 1.84 & 0.10 & 1.45 & 0.129 \\
SM2 & 0.84 & 1.89 & 0.09 & 1.41 & 0.132 \\
SM3 & 1.10 & 2.2 & 0.18 & 1.15 & 0.134 \\
DM1 & 0.91 & 2.03 & 0.12 & 1.24 & 0.136 \\
DM2 & 0.94 & 2.06 & 0.10 & 1.33 & 0.143 \\
DM3 & 0.84 & 1.94 & 0.09 & 1.53 & 0.133 \\
DM4 & 0.86 & 2.02 & 0.09 & 1.47 & 0.137 \\
DM5 & 1.09 & 2.10 & 0.15 & 1.15 & 0.140 \\
Rodi et al. [27] & {$[0.38,1.79]$} & {$[1.66,2.77]$} & {$[0.10,0.27]$} & {$[0.89,2.96]$} & {$[0.07,0.15]$} \\
Sohankar et al. [30] and & {$[1.23,1.54]$} & {$[2.0,2.32]$} & {$[0.16,0.20]$} & {$[1.29-1.34]$} & {$[0.127,0.135]$} \\
Fureby et al. [11] & & 2.1 & - & 1.4 & $0.132 \pm 0.004$ \\
Lyn et al. [21, 22] & - & 2.28 & - & - & 0.130 \\
Bearman and Obasaju [1] & 1.2 & 2.16 & - & - & 0.132 \\
Norberg [24] & - & 2.21 & 0.18 & - & 0.130 \\
Luo et al. [20] & 1.21 & & & &
\end{tabular}

Table 5: Bulk coefficients; comparison with experimental data and with other simulations in the literature. $\overline{C_{d}}$ is the mean drag coefficient, $C_{d}^{\prime}$ and $C_{l}^{\prime}$ are the r.m.s. of the drag and lift coefficients, $S t$ is the Strouhal number and $l_{r}$ is the length of the mean recirculation bubble. 


\begin{tabular}{|c|c|c|c||c|c|c|}
\hline Coefficient & DM1 & DM3 & DM1 vs DM3 & DM2 & DM4 & DM2 vs DM4 \\
\hline \hline $\bar{C}_{d}$ & 2.03 & 1.94 & $-4 \%$ & 2.06 & 2.02 & $-1.9 \%$ \\
\hline$C_{l}^{\prime}$ & 0.91 & 0.84 & $-7.7 \%$ & 0.94 & 0.86 & $-8.5 \%$ \\
\hline$C_{d}^{\prime}$ & 0.12 & 0.09 & $-25 \%$ & 0.10 & 0.09 & $-10 \%$ \\
\hline$l_{r}$ & 1.24 & 1.53 & $+23 \%$ & 1.33 & 1.47 & $+10.5 \%$ \\
\hline
\end{tabular}

Table 6: Influence of $\gamma_{s}$ on results when schemes SC1 (DM1,DM3) and SC2 (DM2,DM4) are used.

The Strouhal number reported in Tab. 5 is defined as follows:

$$
S t=\frac{U_{r e f} D}{f_{s}},
$$

where $U_{r e f}$ is the asymptotic velocity, $D$ is the length of the cylinder side and $f_{s}$ is the shedding frequency measured from the time history of the lift coefficient $C_{l}$. All the simulations predict $S t$ with a good accuracy; thus, this parameter will not be considered in the following discussion.

Results from simulations using the schemes $\mathrm{SC} 1$ and $\mathrm{SC} 2$ are compared in order to evaluate their capabilities when used within an LES approach. The schemes SC1 and SC2 are compared in terms of:

- sensitivity of results to numerical viscosity $\left(\gamma_{s}\right)$;

- accuracy of predictions in comparison with the experiments ;

- sensitivity of results to SGS model ;

Finally, two simulations using the SGS models described in Sec. 2.4 were carried out on a better designed grid on the refined grid (GR2) (SM3 and DM5 of Tab. 4), in order to investigate whether some of the discrepancies with the experiments observed in simulations carried out on on grid GR1 were caused by the inadequacy of the grid, especially in the front part and near the corners of the cylinder.

Numerical viscosity The numerical viscosity has a significant influence on all the bulk coefficients and the agreement with the experiments improves as $\gamma_{s}$ is decreased, as already put in evidence in previous studies ([2],[3]). This behavior can be observed here for both $\mathrm{SC} 1$ and $\mathrm{SC} 2$. When $\mathrm{SC} 1$ is used, it is possible to compare DM1 and DM3 in order to check the influence of $\gamma_{s}$ on the results. Analogously, when SC2 is used, simulations DM2 and DM4 can be considered. The results obtained in these simulations, already shown in Tab. 5 , are reported together in Tab. 6 for sake of clarity. The qualitative variation of the bulk coefficients with $\gamma_{s}$ is the same for both $\mathrm{SC} 1$ and $\mathrm{SC}$; however, all the bulk coefficients, except for $C_{l}^{\prime}$, obtained by SC2 are remarkably less sensitive to $\gamma_{s}$. 


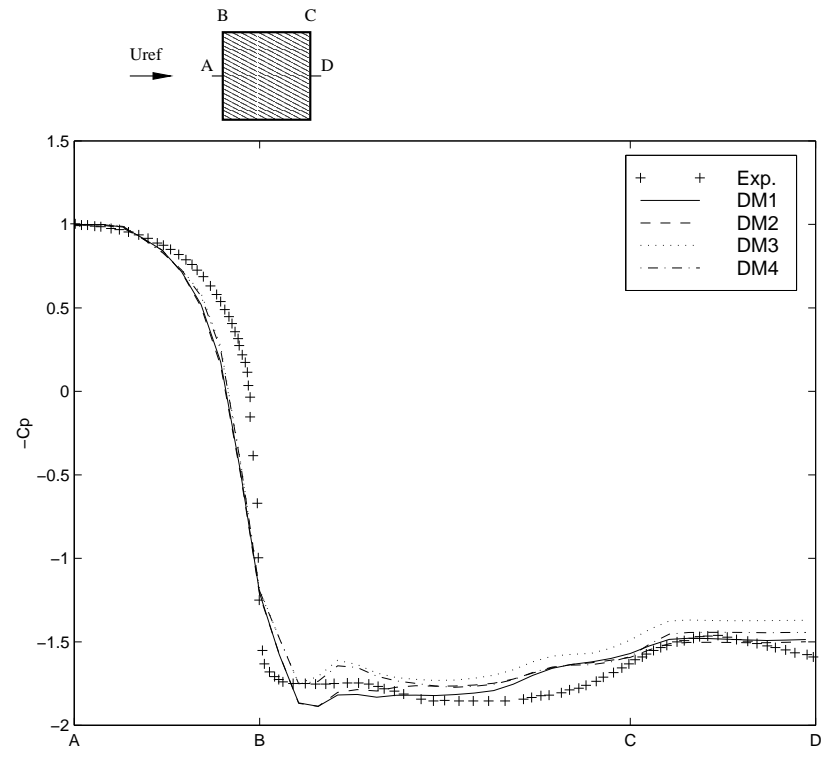

Figure 8: Pressure coefficient on the cylinder surface.

Also, the mean velocity and the pressure fields are less sensitive to $\gamma_{s}$ when SC2 is used. This is shown, for instance, in Fig. 8, where the $\overline{C_{p}}$ distribution over the cylinder surface is reported. As for the mean velocity field, Figs. 9 and 10 show profiles of the streamwise velocity.

Accuracy For a comparison in terms of accuracy between $\mathrm{SC} 1$ and $\mathrm{SC} 2$, it is possible to consider simulations that differ only for the scheme used for the convective fluxes. This is the case for SM1 and SM2, when the Smagorinky model is used, and for DM1, DM2, DM3 and DM4, when the dynamic SGS model is used. The bulk coefficients for these simulations are reported in Tab. 5, while the errors with respect to the experiments are reported in Tab. 7. Tab. 7 shows that the scheme SC2 gives systematically more accurate results than $\mathrm{SC} 1$ for all the bulk coefficients with the exception of $C_{d}^{\prime}$, whose value does not show significant variations in the different simulations considered. Moreover, a comparison between (DM1,DM2) and (DM3,DM4) shows that the advantage of SC2 over SC1 increases as the upwinding of the scheme $\left(\gamma_{s}\right)$ is increased.

This tendency is confirmed by the analysis of time averaged flow fields. The time averaged pressure coefficient $\overline{C_{p}}$ is shown in Fig. 11 for SM1 and SM2, and in Fig. 8 for DM1, DM2, $\mathrm{DM} 3$ and DM4. When $\mathrm{SC} 2$ is used instead, the $\overline{C_{p}}$ accuracy increases on the side and rear faces, especially when $\gamma_{s}=0.1$ (DM3,DM4).

An analogous behavior can be observed from the analysis of time averaged velocity fields, reported in Fig. 12. 

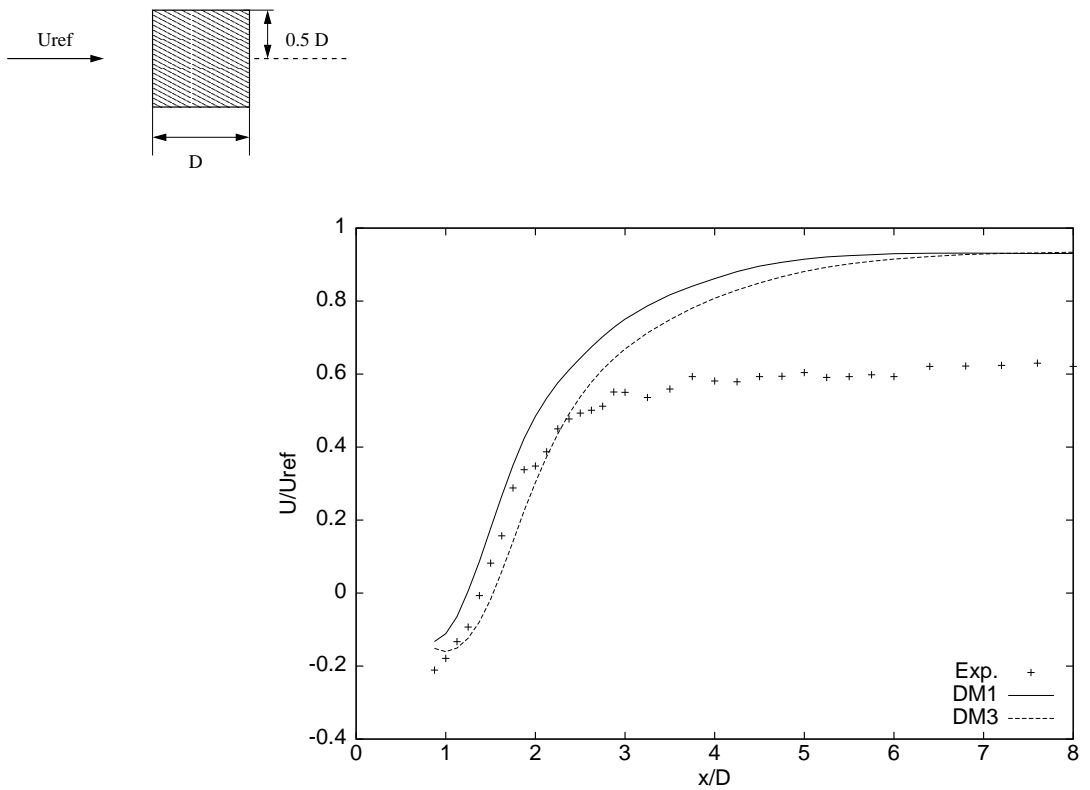

(a) Simulations using SC1

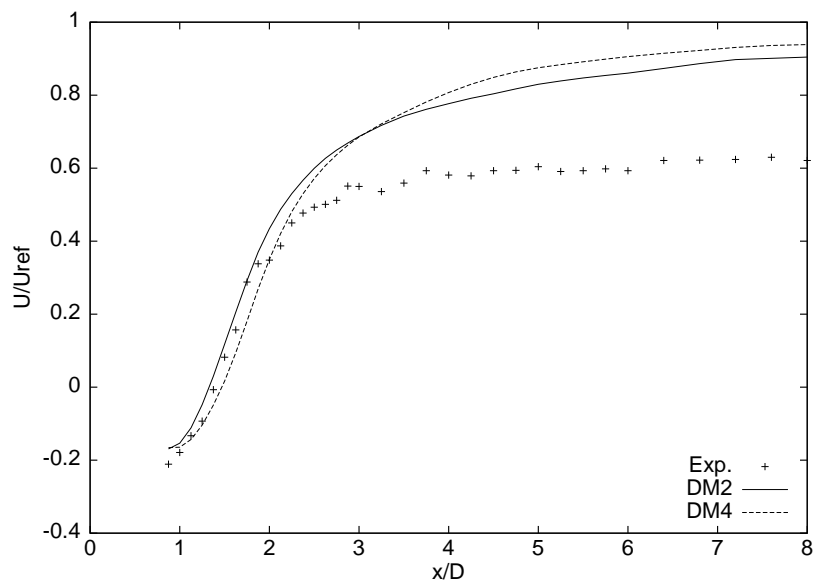

(b) Simulations using SC2

Figure 9: Time averaged velocity profiles obtained using SC1 (a) and SC2 (b) at the section shown at the top of the figure. The profiles are averaged in space in the homogeneous direction.

$\mathrm{RR} \mathrm{n}^{\circ} 4512$ 

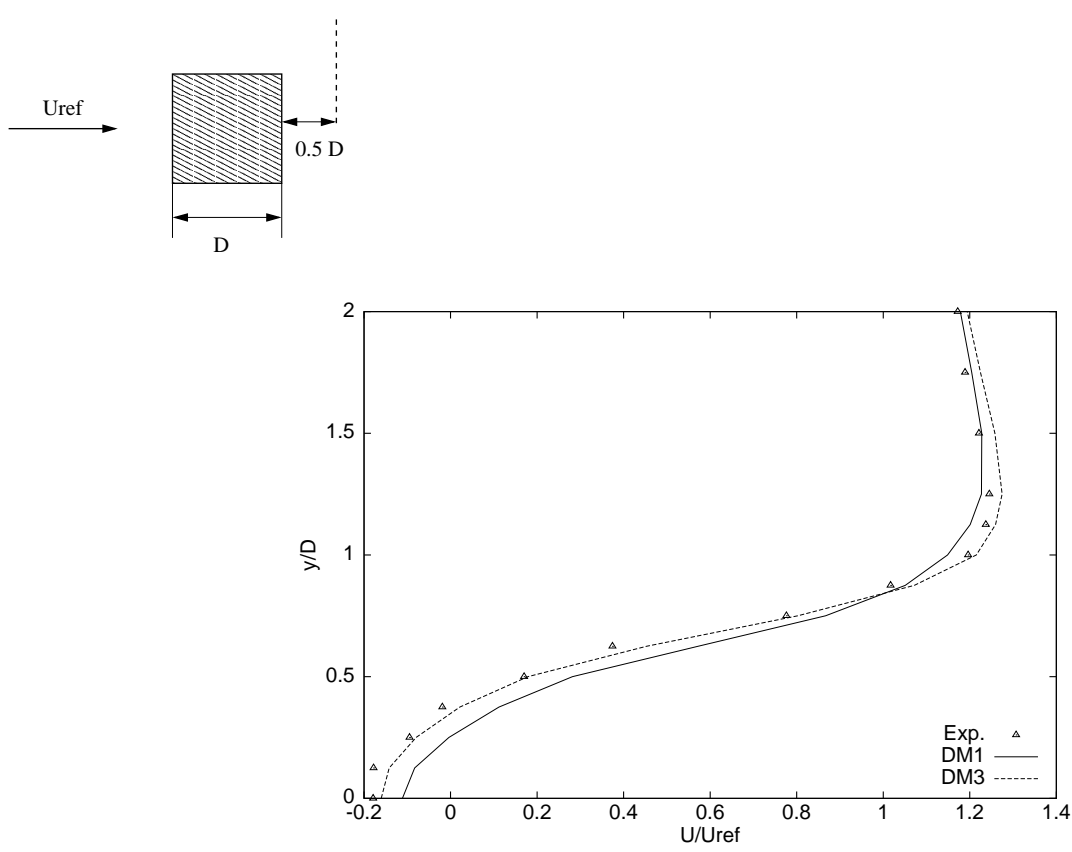

(a) Simulations using SC1

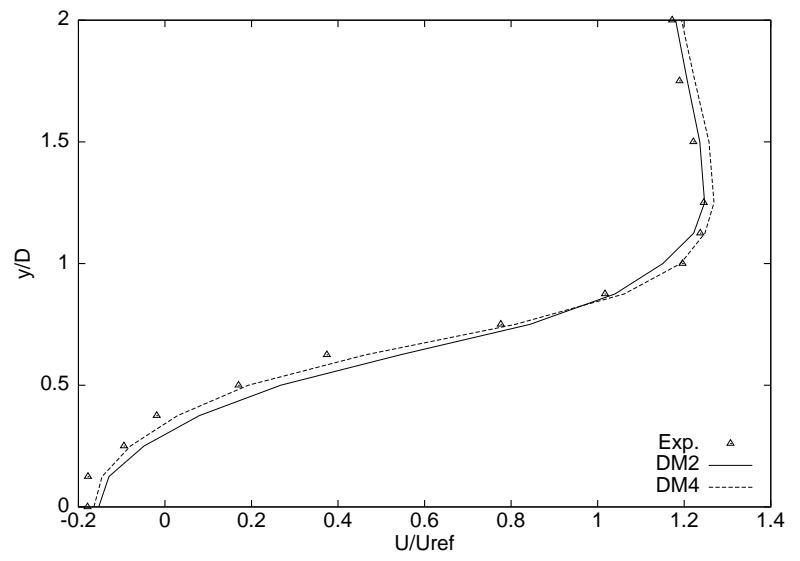

(b) Simulations using SC2

Figure 10: Time averaged velocity profiles obtained using SC1 (a) and SC2 (b) at the section shown at the top of the figure. The profiles are averaged in space in the homogeneous direction. 


\begin{tabular}{c||c|c||c|c||c|c} 
Coefficient & SM1 & SM2 & DM1 & DM2 & DM3 & DM4 \\
\hline$\overline{C_{d}}$ & $-12 \%$ & $-10 \%$ & $-3 \%$ & $-2 \%$ & $-8 \%$ & $-4 \%$ \\
$C_{l}^{\prime}$ & $-34 \%$ & $-30 \%$ & $-24 \%$ & $-21 \%$ & $-30 \%$ & $-28 \%$ \\
$C_{d}^{\prime}$ & $-44 \%$ & $-50 \%$ & $-33 \%$ & $-44 \%$ & $-50 \%$ & $-50 \%$ \\
$l_{r}$ & $+4 \%$ & $+1 \%$ & $-11 \%$ & $-5 \%$ & $+9 \%$ & $+5 \%$ \\
\hline
\end{tabular}

Table 7: Comparison with the experiments. The simulations of the couples (SM1,SM2), (DM1,DM2) and (DM3,DM4) differ only for the scheme used for the convective fluxes (see also Tab. 4). The reference experimental work for $\overline{C_{d}}, l_{r}$ is Ref. [21] and for $C_{l}^{\prime}, C_{d}^{\prime}$ is Ref. [20].

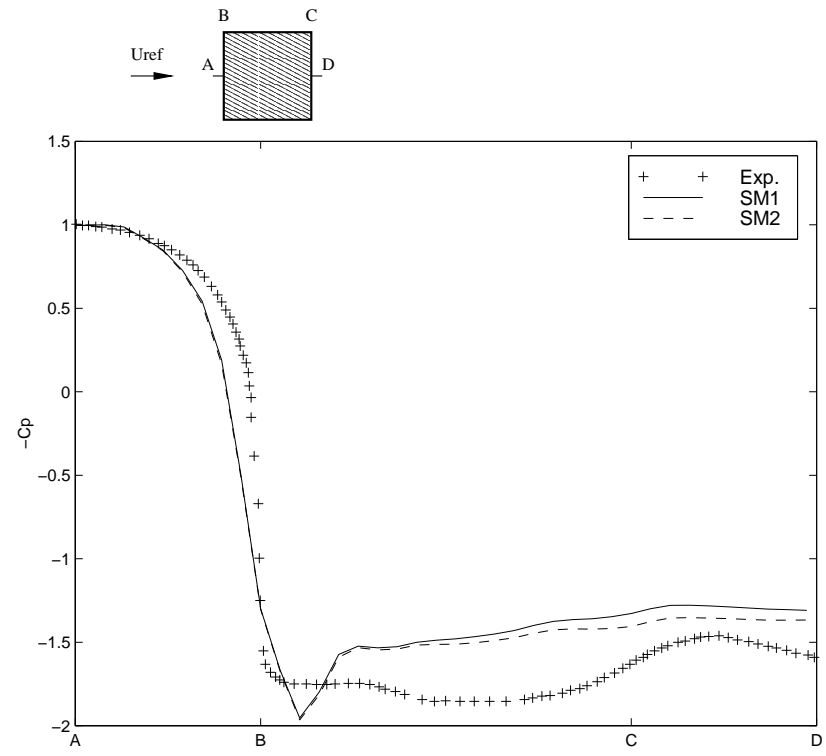

Figure 11: Pressure coefficient on the cylinder surface.

$\mathrm{RR} \mathrm{n}^{\circ} 4512$ 

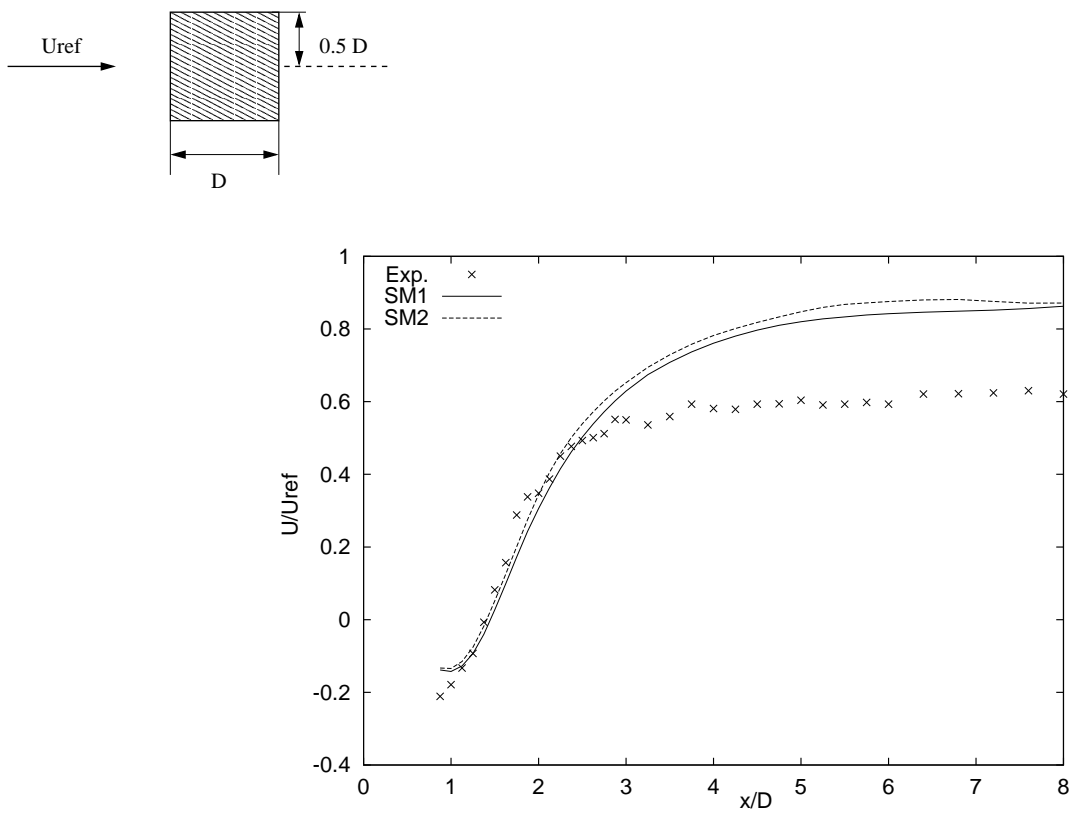

(a) Simulations using SC1

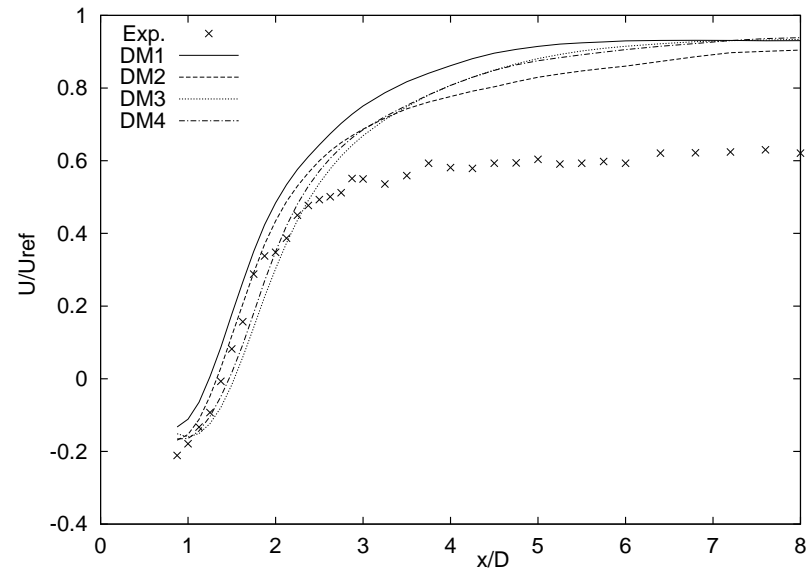

(b) Simulations using SC2

Figure 12: Time averaged velocity profiles at the section shown at the top of the figure. The profiles are averaged in space in the homogeneous direction. 


\begin{tabular}{|c|c|c|c||c|c|c|}
\hline Coefficient & SM1 & DM1 & SM1 vs DM1 & SM2 & DM2 & SM2 vs DM2 \\
\hline \hline$\overline{C_{d}}$ & 1.84 & 2.03 & $+10 \%$ & 1.89 & 2.06 & $+9 \%$ \\
\hline$C_{l}^{\prime}$ & 0.79 & 0.91 & $+15 \%$ & 0.84 & 0.94 & $+11.9 \%$ \\
\hline$C_{d}^{\prime}$ & 0.10 & 0.12 & $+20 \%$ & 0.09 & 0.10 & $+11.1 \%$ \\
\hline$l_{r}$ & 1.45 & 1.24 & $-14.5 \%$ & 1.41 & 1.33 & $-5.7 \%$ \\
\hline
\end{tabular}

Table 8: Influence of SGS model on the results when SC1 (SM1,DM1) and SC2 (SM2,DM2) are used.

SGS model As already discussed in Ref. [3], results of coarse LES are particularly sensitive to the SGS model employed. In our case, the dynamic model generally improves predictions with respect to the Smagorinsky model, as it can be seen in Tab. 5 by comparing SM1 with DM1, SM2 with DM2 and SM3 with DM5.

The point of interest here is to compare $\mathrm{SC} 1$ and $\mathrm{SC} 2$ in terms of sensitivity of the results to the SGS model change. For this purpose, the simulation couples (SM1,DM1) and (SM2,DM2) are considered, where respectively $\mathrm{SC} 1$ and $\mathrm{SC} 2$ is used. The resulting bulk coefficients, already shown in Tab. 5, are reported together in Tab. 8 for sake of clarity. Tab. 8 shows that the qualitative variations of the bulk coefficients with the SGS model are the same for $\mathrm{SC} 1$ and $\mathrm{SC}$. However, quantitatively, the sensitivity of the results obtained with SC2 is lower than with SC1. This is confirmed by the analysis of the time averaged velocity fields, reported for two different sections in Fig. 13 and Fig. 14.

Results shown here prove that the mechanism of interaction between the numerical viscosity and the SGS model is qualitatively the same in SC1 and SC2. However, the different sensitivity of the results to the SGS model could be explained as a a consequence of a reduced interaction effects obtained with SC2. This hypothesis could also justify the gain in accuracy of the results obtained with $\mathrm{SC} 2$ with respect to $\mathrm{SC} 1$. Indeed, it is generally accepted in the literature that the combination of numerical viscosity and the SGS model leads to an excessive dissipation that lowers the results accuracy. In order to show why SC2 could reduce competition between numerics and SGS modeling, it was pointed out in Sec. 2.3 that the scheme SC2 leads to a numerical dissipation that is more localized on high frequencies and proportional to the sixth order derivatives, while the SGS dissipation is proportional to the second-order derivatives. Consequently, $\gamma_{s}$ variations in SC2 affects a more concentrated and limited range of scales than in $\mathrm{SC} 1$ and this could explain the lower sensitivity of results to $\gamma_{s}$ obtained in SC2. Obviously, errors caused by numerical viscosity propagates to all frequency components due to the non-linearity of the equations, and this surely reduces the potential advantages of $\mathrm{SC} 2$ over $\mathrm{SC} 1$ shown by the linear Fourier analysis carried out in Sec. 2.3. However, results obtained in the simulations of Tab. 6 proves that $\mathrm{SC} 2$ is still advantageous over SC1 also in the non linear case.

Simulations on GR2 In the simulations carried out on GR1, a systematic discrepancy with the experiments has been observed in the $\overline{C_{p}}$ distribution on the upwind face $\mathrm{AB}$, which is not dependent from any of the simulations parameters, as it can be seen in Figs. 8 and 11. 

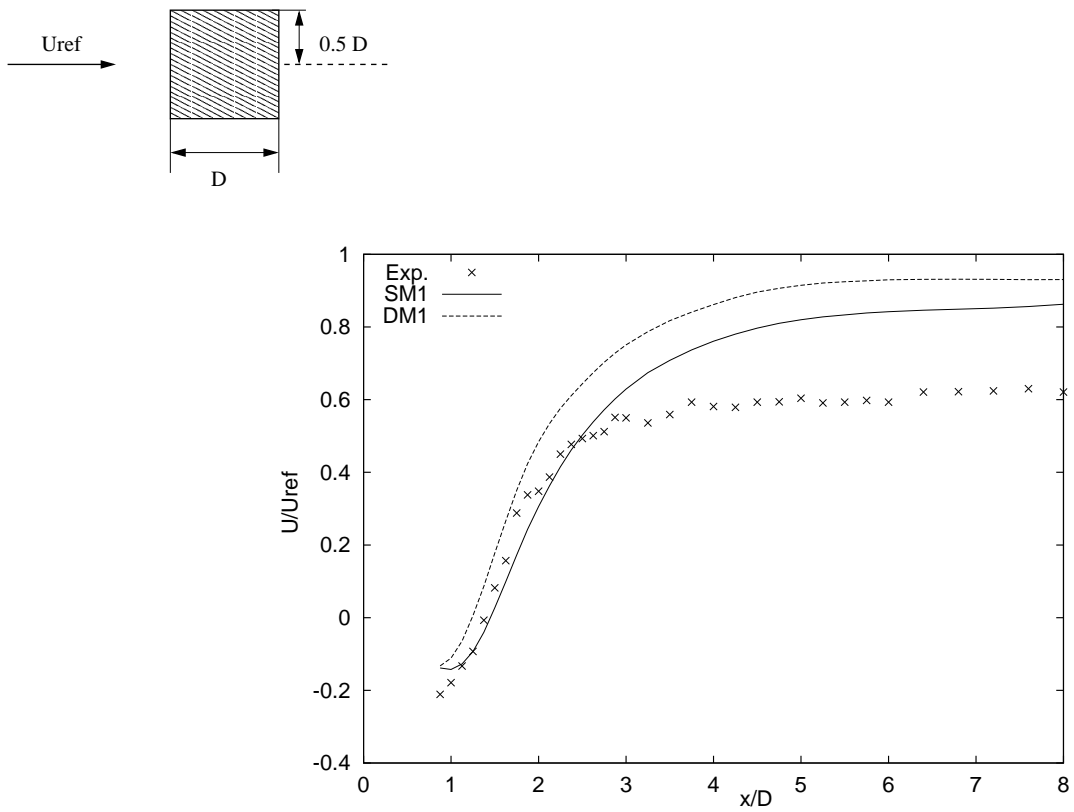

(a) Simulations using SC1

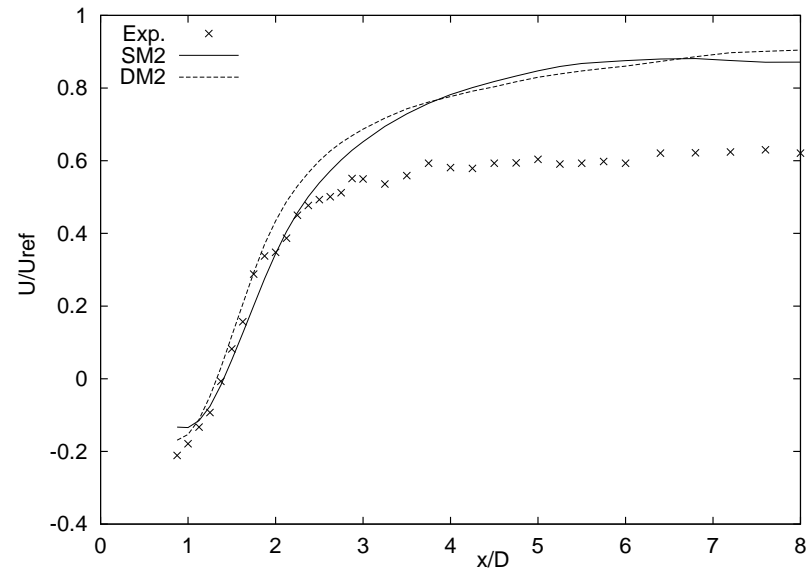

(b) Simulations using SC2

Figure 13: Time averaged velocity profiles at the section shown at the top of the figure. The profiles are averaged in space in the homogeneous direction. 

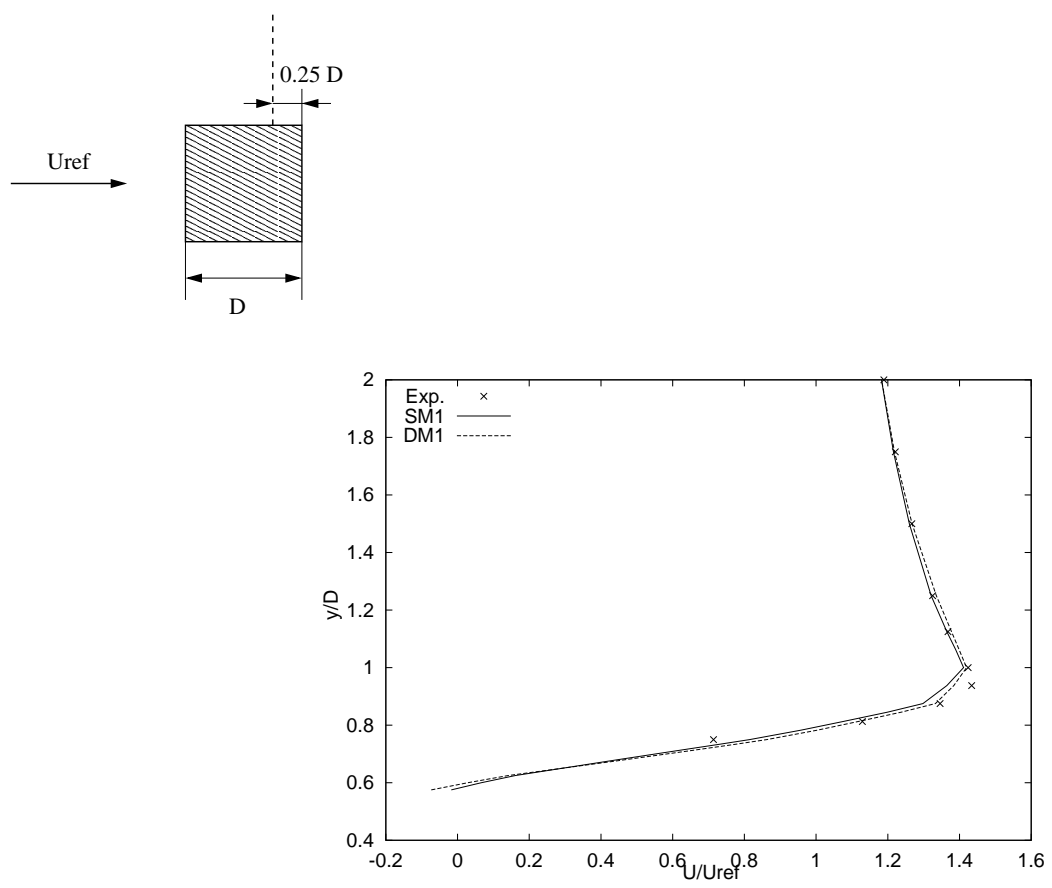

(a) Simulations using SC1

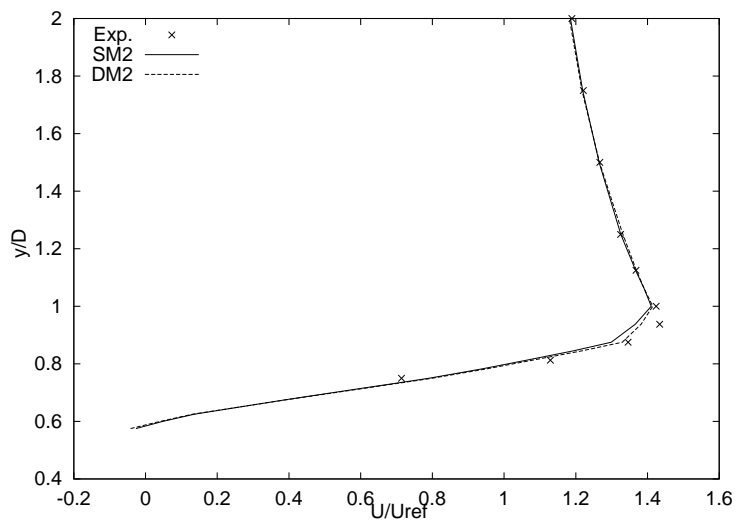

(b) Simulations using SC2

Figure 14: Time averaged velocity profiles at the section shown at the top of the figure. The profiles are averaged in space in the homogeneous direction.

$\mathrm{RR} \mathrm{n}^{\circ} 4512$ 


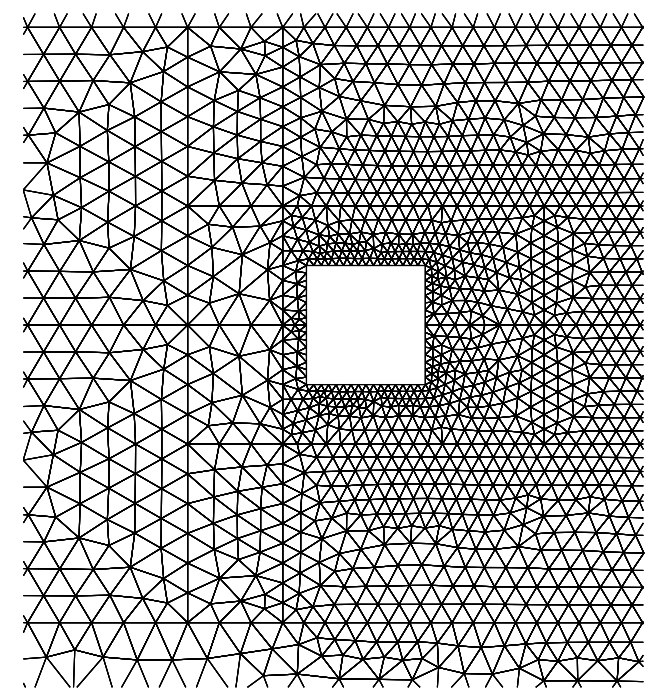

(a) GR1

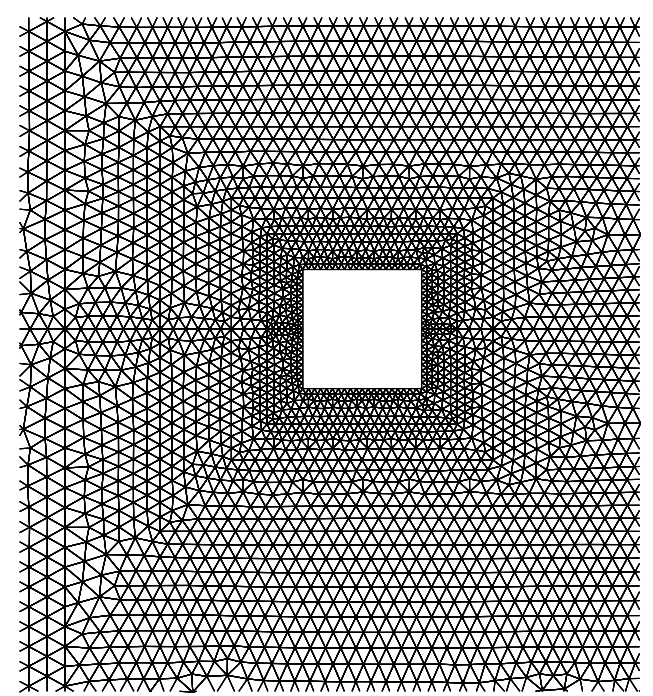

(b) GR2

Figure 15: Node distribution in grids GR1 (a) and GR2 (b) on the symmetry section in spanwise direction.

In particular, the predicted pressure coefficient on face $A B$ is lower than the experimental one and this explains why, even if the base pressure is predicted rather well, the global drag coefficient is still underestimated, as in DM1, DM2 and DM4 (see Tab. 5 and Fig. 8). The grid GR1 is not properly designed near the face AB; indeed, as shown in Fig. 15(a), large elements are located too close to face $\mathrm{AB}$, where gradients are high, and the grid refinement is too sharp to effectively increase resolution. Thus, a better designed grid (GR2), shown in Fig. 15(b), has been used in order to verify if the observed discrepancies indeed depend on the inadequacy of the grid. Two simulations have been carried out on GR2, using the Smagorinsky (SM3) and the dynamic models (DM5) (see Tab. 4). The pressure coefficients obtained on GR2 are reported in Fig. 16, together with the experimental values. The $\overline{C_{p}}$ obtained in both simulations matches the experiments rather well on face $\mathrm{AB}$; however, DM5 encounters problems on the side surface BC, where the time-averaged negative pressure peak is overestimated. Nevertheless, the predicted mean drag coefficient is very close to the experimental value in both cases, as shown in Tab. 5, because the base pressure is well predicted. The same applies for the rms of the lift and drag coefficients, that are in both cases remarkably larger and closer to the experiments than the ones predicted by simulations on GR1. 


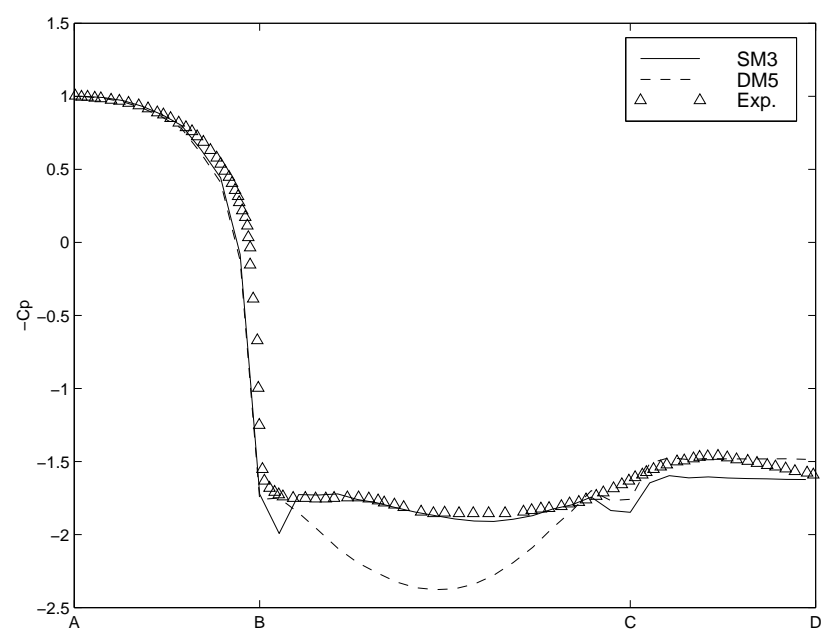

Figure 16: Pressure coefficient on the cylinder surface; simulations on grid GR2.

Concerning the time-averaged velocity field, the recirculation length is severely underpredicted in both SM3 and DM5, as reported in Tab. 5. Indeed, the numerical mean horizontal velocity profile, shown at section $y=0$ (see Fig. 7) in Fig. 17, seems to be translated upwind in $\mathrm{x}$ direction of about a quarter of the cylinder face length in both SM3 and DM5. This happens only in the wake region, while outside the wake predictions are in better accordance with the experiments, as shown in Fig. 18. At the moment, it is difficult to propose an interpretation of this behavior and this point needs further investigation.

As it is possible to notice in Tab. 5, results changes remarkably when GR2 was used instead of GR1, and prediction of the mean bulk coefficients definitely improves, with the exception of the time averaged velocity fields in the wake. Notice that the grid refinement improves results particularly when the Smagorinsky model is used, while the simulations using the dynamic model already gives acceptable results also on the grid GR1. Moreover, the results sensitivity to the SGS model is remarkably reduced when GR2 is used, as it is possible to see by comparing simulations SM3 and DM5. This suggests that, when a coarse grid is used, a dynamic model is to be preferred, even if more expensive, Conversely, for rather resolved simulations with a proper treatment of the boundary conditions at the solid walls, the dynamic model loses its advantages over the Smagorinsky model, at least for what concerns the prediction of global flow quantities, such as bulk coefficients or the time-averaged flow field.

\subsection{CPU time: SC1 vs SC2}

The schemes SC1 and SC2 are now compared in terms of computational cost. Different simulations using both SC1 and SC2 have been performed on grid GR1 to this purpose. The 


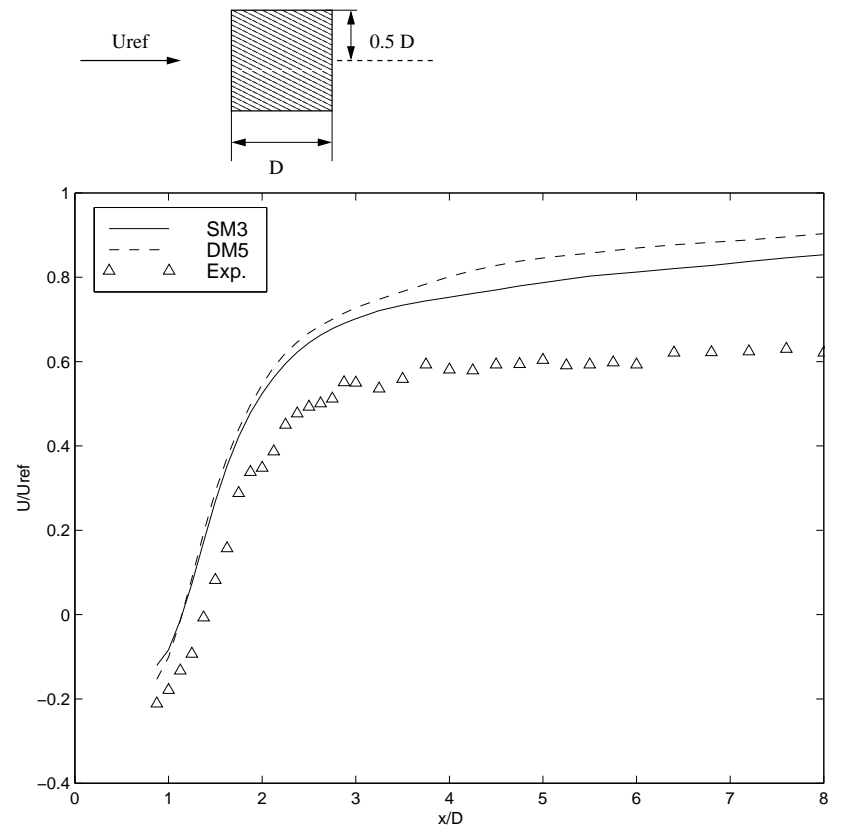

Figure 17: Time averaged velocity profiles obtained in SM3 and DM5 at the section shown at the top of the figure. The profiles are averaged in space in the homogeneous direction. 

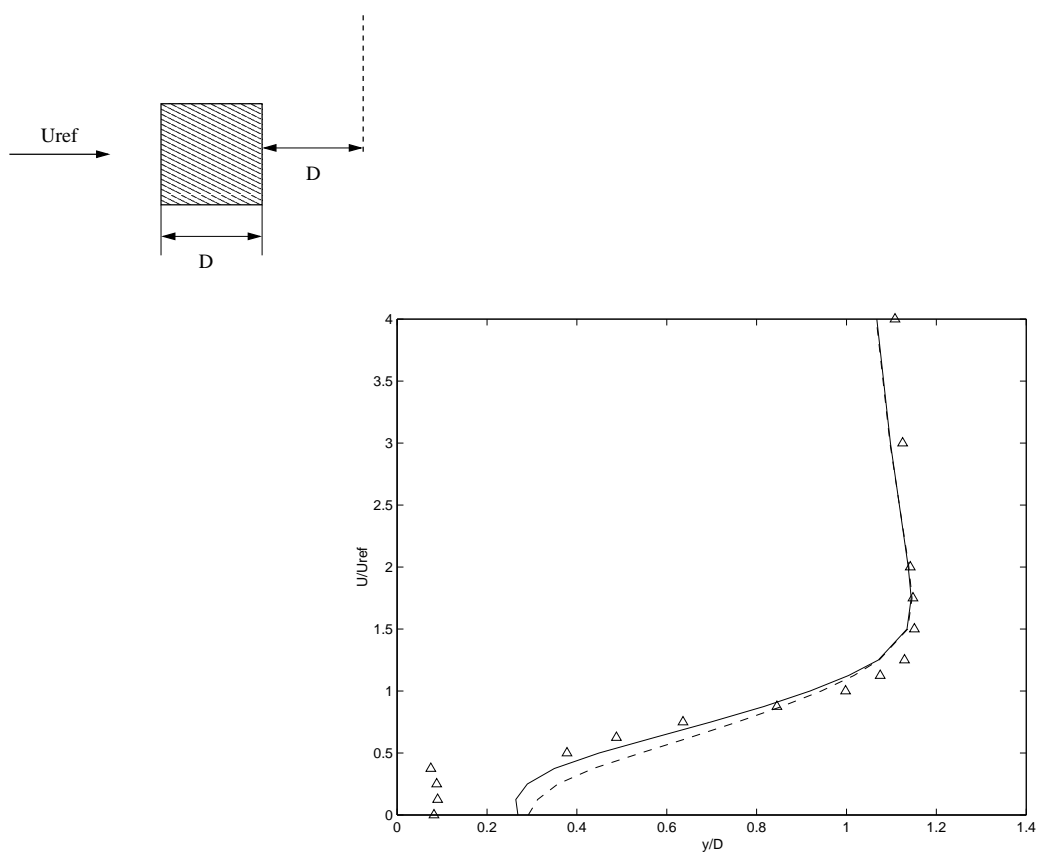

Figure 18: Time averaged velocity profiles obtained in SM3 and DM5 at the section shown at the top of the figure. The profiles are averaged in space in the homogeneous direction.

$\mathrm{RR} \mathrm{n}^{\circ} 4512$ 
explicit 4-stage Runge-Kutta algorithm R1 (see Tab. 2) has been used to advance the simulations in time. The necessary CPU time for each simulation has been evaluated as an average over 100 time iterations. The simulations performed are reported in Tab. 9. Simulations

\begin{tabular}{|c|c|c|c|}
\hline Simulation & Scheme for convective fluxes & SGS model & Periodicity \\
\hline CPU-1 & SC1 & Smag. & NO \\
\hline CPU-2 & SC2 & Smag. & NO \\
\hline CPU-3 & SC1 & Smag. & YES \\
\hline CPU-4 & SC2 & Smag. & YES \\
\hline CPU-5 & SC1 & Dyn. & YES \\
\hline CPU-6 & SC2 & Dyn. & YES \\
\hline
\end{tabular}

Table 9: Simulations performed in order to compare schemes SC1 and SC2 in terms of computational cost

CPU-1, CPU-3 and CPU-5 can be compared respectively with CPU-2 (Smagorinky model), CPU-4 (Smagorinsky model, periodic boundary conditions) and CPU-6 (Dynamic model, periodic boundary conditions). The two simulations of each couple have been performed using respectively the schemes SC1 and SC2 (see Tab. 9).

The increments of total CPU time when SC2 scheme is used instead of SC1, are reported (in percents) in Tab. 10. We have also measured that scheme $\mathrm{SC} 2$ requires about $100 \%$ more CPU time than scheme $\mathrm{SC} 1$ for the discretization of the convective fluxes. This yields an increase of less than $60 \%$ of total CPU time when the Smagorinsky modes is used, independently of the boundary conditions. However, this increment is halved when the dynamic SGS model is used, indicating that the increase of CPU time due to the present implementation of the dynamic SGS model is higher than the one due to the scheme SC2. The global increment of CPU time for SC2 is even much smaller (10\%) when an implicit advancing relying only on a first order operator is applied.

We conclude that changing from scheme $\mathrm{SC} 1$ to scheme $\mathrm{SC} 2$ has a rather small impact on $\mathrm{CPU}$ and is a priori much less CPU consuming than an extension to a fully higher order accurate approximation.

\begin{tabular}{|c|c|c|c|}
\hline & CPU-1/CPU-2 & CPU-3/CPU-4 & CPU-5/CPU-6 \\
\hline Total CPU increment & $57 \%$ & $56 \%$ & $23 \%$ \\
\hline
\end{tabular}

Table 10: Increments in CPU time required when SC2 is used instead of SC1. 


\section{Conclusions}

The present work is a part of a wider research activity ([2], [3]) dedicated to the mutual adaption of a LES approach to turbulence with an industrial CFD methodology. As a demonstrator of this methodology, we have considered a numerical solver (AERO) that is based on a vertex centered finite-volume/finite-element formulation. Since this scheme is of co-located type, approximate Riemann solvers of Roe combined with the MUSCL interpolation are considered for spatial stability. The resulting schemes here applied are second-order accurate and they contain an upwinding parameter, $\gamma_{s}$, which directly controls the numerical diffusion. Previous investigations [3] have been carried out using the convective fluxes scheme SC1, containing a numerical viscosity made of fourth-order derivatives. In the present work we have considered and tested a new scheme for the discretization of the convective fluxes. proposed in Ref. [7], that gives a numerical diffusion based on sixth-order derivatives. Since the SGS dissipation is proportional to the second-order derivatives, this new scheme (SC2) should further enhance the complementarity between the SGS model and the MUSCL stabilization and it should reduce their competition with respect to SC1. This has been confirmed by a preliminary linear analysis of the schemes in which it has been shown that, when scheme $\mathrm{SC} 2$ is used instead of $\mathrm{SC}$, the numerical viscosity action is more localized on high frequency components, that are polluted by elevated aliasing errors.

The simulation of the flow around a square cylinder at $R e=22000$ has been considered in order to test the schemes SC1 and SC2. Experimental ([1],[20],[21],[22],[24]) and numerical LES ([11],[27],[30]) results are available in the literature. Moreover, previous simulations using $\mathrm{AERO}$ and $\mathrm{SC} 1$ for the same flow are documented in [3]. In the present paper, simulations are carried out on a coarse grid (GR1) made of around $10^{5}$ nodes, using both SC1 and SC2 and varying the upwinding parameter $\gamma_{s}$ and the SGS model (Smagorinsky model (Sec. 2.4.1) and its dynamic version (Sec. 2.4.2)). Variations of the results to the simulation parameters are qualitatively the same when $\mathrm{SC} 1$ or $\mathrm{SC} 2$ are used, indicating that the basic mechanism of interaction between numerical and SGS viscosities has not changed. However, when SC2 is used, results are less sensitive to variations of $\gamma_{s}$, of mesh, and, to some extend to the SGS model employed. They are generally more accurate. This seems to confirm our explanation that competition between SGS and numerical viscosities has been reduced with $\mathrm{SC}$, as expected from the linear analysis.

Acknowledgement : The computations presented here were performed at Centre Charles Hermite computing center at Nancy.

$\mathrm{RR} \mathrm{n}^{\circ} 4512$ 


\section{References}

[1] PW. Bearman and ED. Obasaju. An experimental study of pressure fluctuations on fixed and oscillating square-section cylinders. J. Fluid Mech., 119:297-321, 1981.

[2] S. Camarri and M.V. Salvetti. Towards the large-eddy simulation of complex engineering flows with unstructured grids. Technical Report RR-3844, INRIA, 1999.

[3] S. Camarri, M.V. Salvetti, B. Koobus, and A. Dervieux. Large-eddy simulation of a bluff-body flow on unstructured grids. submitted for publication, 2001.

[4] F. Chalot, B. Marquez, M. Ravachol, F. Ducros, F. Nicoud, and Th. Poinsot. A consistent finite element approach to large eddy simulation. In AIAA Paper 98-2652, 1998.

[5] O. Colin, F. Ducros, D. Veynante, and T. Poinsot. A thickened flame model for largeeddy simulations of turbulent premixed combustion. Phys. Fluids, 12:1843-1863, 2000.

[6] O. Colin and M. Rudgyard. Development of high-order taylor-galerkin schemes for les. J. Comp. Phys., 162:338-371, 2000.

[7] C. Debiez and A. Dervieux. Mixed element volume muscl methods with weak viscosity for steady and unsteady flow calculation. Computers and Fluids, 29:89-118, 2000.

[8] F. Ducros, F. Nicoud, and T. Schönfeld. Large-eddy simulations of compressible flows on hybrid meshes. In Proc. of 11th Symp. Turbulent Shear Flows, pages 28-1, Grenoble (France), 1997.

[9] G. Erlebacher, M.Y. Hussaini, C.G. Speziale, and T.A. Zang. Toward the large-eddy simulation of compressible flows. J. Fluid Mech., 238:155-185, 1992.

[10] C. Farhat, B. Koobus, and H. Tran. Simulation of vortex shedding dominated flows past rigid and flexible structures. In Computational Methods for Fluid-Structure Interaction, pages 1-30. Tapir, 1999.

[11] C. Fureby, G. Tabor, HG. Weller, and AD. Gosman. Large eddy simulation of the flow around a square prism. AIAA Journal, 38(3):442-452, 2000.

[12] E. Garnier, P. Sagaut, P. Comte, and M. Deville. On the use of shock-capturing schemes for large-eddy simulation. J. Comp. Phys, 153:273-311, 1999.

[13] M. Germano, U. Piomelli, P. Moin, and W. Cabot. A dynamic subgrid-scale eddy viscosity model. Phys. Fluids A, 3(7):1760-1765, 1991.

[14] D.C. Haworth and K.E. Jansen. Large-eddy simulation on unstructured deforming meshes: towards reciprocating ic engines. Computers and Fluids, 29:493-524, 2000.

[15] K.E. Jansen. Large-eddy simulations of flow around a naca 4412 airfoil using unstructured grids. Annual research briefs, CTR, 1996. 
[16] K.E. Jansen. A stabilized finite element method for computing turbulence. Computer Meth. Appl. Mech. Eng., 174(3-4):299-317, 1999.

[17] R. Lardat, R. Carpentier, B. Koobus, E. Schall, A. Dervieux, C. Farhat, J.F. Guery, and P. Della Pieta. Interaction between a pulsating flow and a perforated membrane. Revue Europeenne des Elements Finis, 9(6-7):805-817, 2000.

[18] R. Lardat, B. Koobus, E. Schall, A. Dervieux, and C. Farhat. Aeroelastic coupling between a thin divergent and high pressure jets. Revue Europeenne des Elements Finis, $9(6-7): 835-851,2000$.

[19] M. Lesieur and P. Comte. Large-eddy simulations of compressible turbulent flows. Technical Report R-819, AGARD, 1997.

[20] SC. Luo, MdG. Yazdani, YT. Chew, and TS. Lee. Effects of incidence and afterbody shape on flow past bluff cylinders. J. Ind. Aerodyn. Ind. Aerodyn., 53:375-399, 1994.

[21] D.A. Lyn, S. Einav, W. Rodi, and J.H. Park. A laser-doppler velocimeter study of ensemble-averaged characteristics of the turbulent near wake of a square cylinder. $J$. Fluid Mech., 304:285-319, 1995.

[22] DA. Lyn and W. Rodi. The flapping shear layer formed by flow separation from the forward corner of a square cylinder. J. Fluid Mech., 267:353-376, 1993.

[23] F. Nicoud and F. Ducros. Subgrid-scale stress modelling based on the square of the velocity gradient tensor. Flow Turb. Comb., 62(3), 1999.

[24] C. Norberg. Flow around rectangular cylinders: pressure forces and wake frequencies. J. Wind Eng. Ind. Aerodyn., 49:187-196, 1993.

[25] N. Okong'o, D. Knight, and G. Zhou. Large eddy simulations using an unstructured grid compressible navier-stokes algorithm. Int. J. CFD, 13:303-326, 2000.

[26] R. Martin R and H. Guillard. A second-order defect correction scheme for unsteady problems. Comput. and Fluids, 25:9-27, 1996.

[27] W. Rodi, J.H. Ferziger, M. Breuer, and M. Pourquié. Status of large eddy simulation: results of a workshop. ASME J. Fluids Eng., 119:248-262, 1997.

[28] PL. Roe. Approximate riemann solvers, parameters, vectors and difference schemes. J. Comp. Phys., 43:357-372, 1981.

[29] E. Schall, R. Lardat A., Dervieux, B. Koobus, and C. Farhat. Aeroelastic coupling between a thin divergent and high pressure jets. Revue Europeenne des Elements Finis, $9(6-7): 835-851,2000$. 
[30] A. Sohankar, L. Davidson, and C. Norberg. Large eddy simulation of flow past a square cylinder: comparison of different subgrid scale models. ASME J. Fluids Eng., 122:39-47, 2000.

[31] J.L. Steger and R.F. Warming. Flux vector splitting for the inviscid gas dynamic equations with applications to finite difference methods. J. Comp. Phys., 40:263-293, 1981.

[32] B. van Leer. Towards the ultimate conservative difference scheme i. the quest of monotonicity. Lecture notes in Physics, 18:163, 1972.

INRIA 


\section{APPENDIX I}

The stability of the schemes of Tab. 1, when used together with the Runge Kutta algorithms of Tab. 2, is appraised here by performing tests directly on the code. The turbulent channel flow test case has been selected to this purpose. The computational domain is sketched in Fig. 19. With reference to Fig. 19, periodic boundary conditions are imposed in streamwise $(X)$ and spanwise $(Y)$ direction, while no-slip conditions are imposed on the solid walls $(Z=0, Z=-4 / 3 \pi)$. A coarse grid of about $4 \cdot 10^{4}$ nodes was used to simulate the flow with

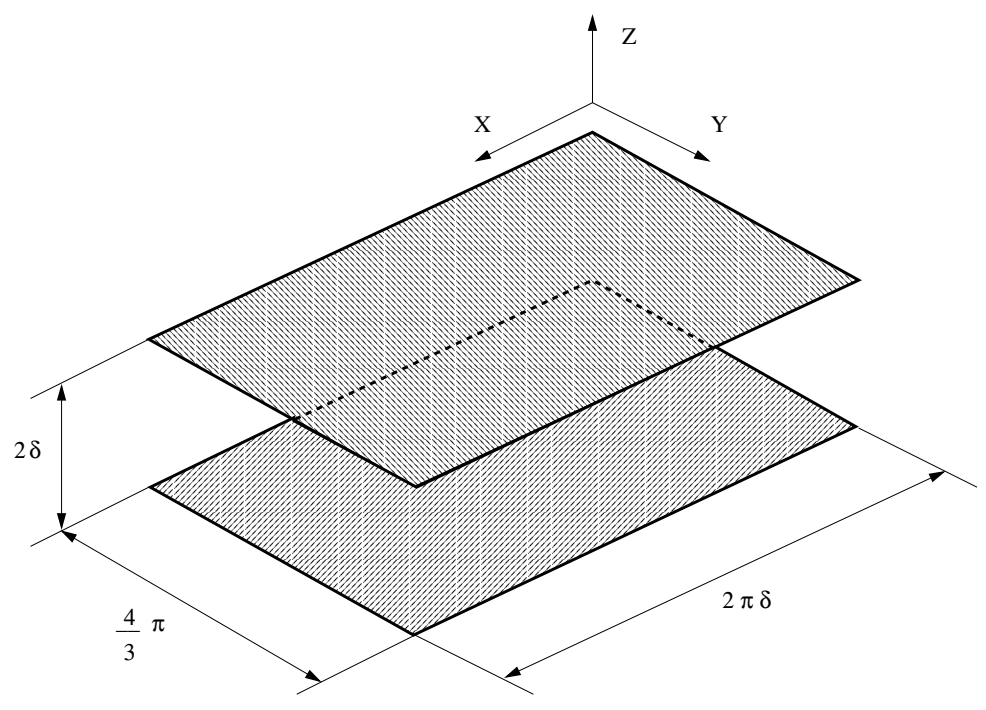

Figure 19: Turbulent channel flow test case: computational domain

a wall Reynolds number $R e_{\tau}=180$. Schemes of Tab. 1 and Tab. 2 have been combined together and, for each combination, the maximum CFL number for numerical stability has been searched by carrying out a short simulation. Results are reported in Tab. 11. Results

\begin{tabular}{|c|c|c|c|}
\hline & R1 & R2 & R3 \\
\hline SC1 & 2.1 & 2.3 & 1.5 \\
SC2 & 2.0 & 2.1 & 1.5 \\
SC3 & 1.9 & 2.0 & 1.5 \\
SC4 & $<0.8$ & $<0.8$ & 1.0 \\
\hline
\end{tabular}

Table 11: CFL max for numerical stability when schemes in Tab. 1 are used in combination with the Runge-Kutta algorithms in Tab. 2.

reported in Tab. 11 show that R1 is the stablest Runge-Kutta algorithm when used together with the V6 schemes. While the schemes R2 has a behavior similar to R1, the scheme R3 is 
remarkably less stable. The only exception concerns the scheme SC4, for which neither R1 or R2 give enough stability. On the contrary, when SC4 is coupled with R3, a stable scheme with $C F L_{\max }=1$ is obtained. Although the combination SC4-R3 is noticeably less stable than the others of Tab. 11, it has the advantage of being 6-th order accurate in space (see Tab. 1), as discussed in Sec. 2.1.3.

INRIA 


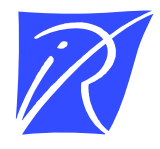

Unité de recherche INRIA Sophia Antipolis 2004, route des Lucioles - BP 93 - 06902 Sophia Antipolis Cedex (France)

Unité de recherche INRIA Lorraine : LORIA, Technopôle de Nancy-Brabois - Campus scientifique 615, rue du Jardin Botanique - BP 101 - 54602 Villers-lès-Nancy Cedex (France)

Unité de recherche INRIA Rennes : IRISA, Campus universitaire de Beaulieu - 35042 Rennes Cedex (France)

Unité de recherche INRIA Rhône-Alpes : 655, avenue de l'Europe - 38330 Montbonnot-St-Martin (France)

Unité de recherche INRIA Rocquencourt : Domaine de Voluceau - Rocquencourt - BP 105 - 78153 Le Chesnay Cedex (France)

Éditeur

INRIA - Domaine de Voluceau - Rocquencourt, BP 105 - 78153 Le Chesnay Cedex (France)

http://www.inria.fr

ISSN 0249-6399 